\title{
On Robust Stability of Stochastic Genetic Regulatory Networks With Time Delays: A Delay Fractioning Approach
}

\author{
Yao Wang, Zidong Wang, Senior Member, IEEE, and Jinling Liang
}

\begin{abstract}
Robust stability serves as an important regulation mechanism in system biology and synthetic biology. In this paper, the robust stability analysis problem is investigated for a class of nonlinear delayed genetic regulatory networks with parameter uncertainties and stochastic perturbations. The nonlinear function describing the feedback regulation satisfies the sector condition, the time delays exist in both translation and feedback regulation processes, and the state-dependent Brownian motions are introduced to reflect the inherent intrinsic and extrinsic noise perturbations. The purpose of the addressed stability analysis problem is to establish some easy-to-verify conditions under which the dynamics of the true concentrations of the messenger ribonucleic acid (mRNA) and protein is asymptotically stable irrespective of the norm-bounded modeling errors. By utilizing a new Lyapunov functional based on the idea of "delay fractioning", we employ the linear matrix inequality (LMI) technique to derive delay-dependent sufficient conditions ensuring the robust stability of the gene regulatory networks. Note that the obtained results are formulated in terms of LMIs that can easily be solved using standard software packages. Simulation examples are exploited to illustrate the effectiveness of the proposed design procedures.
\end{abstract}

Index Terms-Genetic regulatory networks (GRNs), linear matrix inequality (LMI), Lyapunov-Krasovskii functional, robust stability, stochastic perturbation, time delays, uncertain system.

\section{INTRODUCTION}

$\mathbf{T}$ HE PAST few years have witnessed the significant progress in the research area of gene engineering and other biological sciences. The mechanisms that have evolved to regulate the gene expression are known as genetic regulatory networks (GRNs). With the study of GRNs, scientists would be

Manuscript received March 2, 2009; revised May 31, 2009. This work was supported in part by the Biotechnology and Biological Sciences Research Council of U.K. under Grant BB/C506264/1, by an International Joint Project sponsored by the Royal Society of U.K., by the National Natural Science Foundation of China under Grant 60804028, by the Teaching and Research Fund for Excellent Young Teachers at Southeast University of China, by the Specialized Research Fund for the Doctoral Program of Higher Education for New Teachers in China under Grant 200802861044, and by the Alexander von Humboldt Foundation of Germany. This paper was recommended by Associate Editor H. Gao.

Y. Wang is with the School of Information Science and Technology, Donghua University, Shanghai 200051, China (e-mail: enidyao@gmail.com).

Z. Wang is with the Department of Information Systems and Computing, Brunel University, UB8 3PH Uxbridge, U.K. (e-mail: Zidong.Wang@ brunel.ac.uk).

J. Liang is with the Department of Mathematics, Southeast University, Nanjing 210096, China (e-mail: jinlliang@gmail.com).

Color versions of one or more of the figures in this paper are available online at http://ieeexplore.ieee.org.

Digital Object Identifier 10.1109/TSMCB.2009.2026059 able to explain the interactions between genes and protein that form complex biological systems. It is of great importance to investigate and understand the gene regulatory process and the dynamic behaviors of the GRNs in living organisms [1], [2], [4], [7], [27].

The modeling of GRNs is largely dependent on powerful tools of mathematics theory. In general, the GRNs can be described by two types of models, i.e., the discrete model (such as Boolean networks) and the continuous model (such as the differential equation model) [1], [7], [8], [21], [32]. Recently, among all the proposed GRN models, the differential equation models have received an increasing amount of research attention since the variables in gene dynamics are usually the concentrations of gene products (i.e., messenger ribonucleic acids (mRNAs) and proteins), which possess continuous values of the genetic regulatory systems (see [2], [3], [13], [14], [20], [27], [28], and the references therein). Our present research further examines the continuous GRN models with both time delays and norm-bounded parameter uncertainties.

It is well known that the existence of time delays is ubiquitous in biological, physical, chemical, and electrical dynamical systems [9]. In biological systems, particularly GRNs, time delays are unavoidable primarily due to the finite speed in the slow process of transcription, translation, and translocation. It has been shown in [13] and [14] that the time delays in GRNs may play an important role in the predictions of the dynamics of the mRNA and protein concentrations. Moreover, given the facts that GRNs are modeled from real-world gene expression time-series data, and that there are certain limitations with the current experimental techniques, it has now been well recognized that the modeling errors and parameter fluctuations are inevitable, which may cause poor performance or even instability of real genetic networks [2], [4], [10]. It should be pointed out that the system parameters identified from experimental data may form an unknown but bounded time-varying function (see [20] and [30]). When investigating the dynamical behaviors of GRNs, the parameter uncertainties (also called variations or fluctuations) should also be taken into account, and therefore, the stability robustness issue for GRNs emerges as a research topic of great importance.

On the other hand, the modeling of GRNs should be conducted in a way to interpret vast amounts of experimental data and the extracted functional information from observation data. Given the fact that biology networks or genetic networks are always subject to random fluctuations [1], [4], [18], [22], 
[23], it is vitally important to consider the random effects including both the intrinsic and extrinsic noise perturbations [1], [14], [27], [29]. Both the modeling error and the stochastic disturbances could cause instability [29] of the networks and make it difficult to know the true dynamics of the network state. Therefore, an interesting problem of biological significance is to investigate the robust stochastic stability in the presence of time delays, parameter uncertainties, and stochastic disturbances for the addressed GRNs, which give rise to another motivation for the present research.

Although the robust stability of GRNs has stirred some initial research interests [2], [4], [20], one of the main issues aroused here is how to reduce the possible conservatism induced by the introduction of the Lyapunov functional when dealing with time delays, which leaves much room for further research by using the latest analysis techniques. Recently, the so-called "delay fractioning" approach, which is arguably the up-to-date delay-dependence analysis method, has independently been originated from [12] and [19] and further developed in [16], [17], [26], [33], and [35], and shown to lead to much less conservative results than most existing literature. It is, therefore, the main purpose of this paper to adopt the delay-fractioning approach for achieving a less conservative delay-dependence condition to guarantee the robust stability of the addressed GRNs.

In this paper, we are concerned with the robust stability analysis problem for a class of uncertain GRNs with and without noise perturbations, where the time delays exist in both the translation process and the feedback regulation process, and the nonlinear function describing the feedback regulation is assumed to satisfy the sector condition. By utilizing a novel Lyapunov-Krasovskii functional and the linear matrix inequality (LMI) technique, sufficient delay-dependent conditions ensuring the robust stability of the gene regulatory model are established. The obtained results are formulated in the form of LMIs that are easily solvable by using standard software packages. Simulation examples with three component genetic networks are used to illustrate the effectiveness of the developed theoretical results.

Notations: The notations used throughout this paper are fairly standard. $\mathfrak{R}^{n}$ and $\mathfrak{R}^{n \times m}$ denote the $n$-dimensional Euclidean space and the set of $n \times m$ real matrices, respectively, and $|\cdot|$ is the Euclidean norm on $\mathfrak{R}^{n} . P>0$ means that matrix $P$ is real, symmetric, and positive definite. $I$ and 0 denote the identity matrix and the zero matrix with compatible dimensions, respectively, $\operatorname{diag}\{\cdots\}$ stands for a block-diagonal matrix, and $\operatorname{col}\{\cdots\}$ denotes a matrix column with blocks given by the matrices in $\{\cdots\}$. The superscript " $T$ " stands for matrix transposition, and the asterisk "*" in a matrix is used to represent the term that is induced by symmetry. The Kronecker product of matrices $Q \in \mathbb{R}^{m \times n}$ and $R \in \mathbb{R}^{p \times q}$ is a matrix in $\mathbb{R}^{m p \times n q}$ and denoted as $Q \otimes R$. Moreover, let $\left(\Omega, \mathcal{F},\left\{\mathcal{F}_{t}\right\}_{t \geq 0}, \mathcal{P}\right)$ be a complete probability space with a filtration $\left\{\mathcal{F}_{t}\right\}_{t>0}$ satisfying the usual conditions (i.e., the filtration contains all $\mathcal{P}$-null sets and is right continuous). Denote by $L_{\mathcal{F}_{0}}^{p}\left([-h, 0] ; \mathbb{R}^{n}\right)$ the family of all $\mathcal{F}_{0}$-measurable $\mathcal{C}\left([-h, 0] ; \mathbb{R}^{n}\right)$-valued random variables $\xi=$ $\{\xi(\theta):-h \leq \theta \leq 0\}$ such that $\sup _{-h<\theta<0} \mathbb{E}\left\{|\xi(\theta)|^{p}\right\}<\infty$, where $\mathbb{E}\{\cdot\}$ stands for the mathematical expectation operator with respect to the given probability measure $\mathcal{P}$. Matrix dimensions, if they are not explicitly stated, are assumed to be compatible for algebraic operations.

\section{Model Description ANd Preliminaries}

In this paper, we consider a GRN with time delays existing in both the translation process and the feedback regulation process, which can be described by the following differential equations:

$$
\Sigma:\left\{\begin{array}{l}
\frac{d m_{i}(t)}{d t}=-a_{i} m_{i}(t)+\sum_{j=1}^{n} b_{i j} f_{j}\left(p_{j}(t-\sigma)\right)+\eta_{i} \\
\frac{d p_{i}(t)}{d t}=-c_{i} p_{i}(t)+d_{i} m_{i}(t-\tau)
\end{array}\right.
$$

where $i=1,2, \ldots, n, m_{i}(t), p_{i}(t) \in \mathfrak{R}$ denote, respectively, the concentrations of mRNA and protein of the $i$ th gene at time $t, a_{i}$ and $c_{i}$ are the degradation rates of mRNA and protein of the $i$ th gene, respectively, $d_{i}$ represents the translation rate, $f_{j}\left(p_{j}(s)\right)$ denotes the feedback regulation of the protein on the transcription, which is generally a nonlinear function with monotonicity and satisfies certain conditions given later, the two positive scalars $\tau$ and $\sigma$ denote, respectively, the translation time delay and the feedback regulation delay, $\eta_{i}$ is the base transcriptional rate of the repressor of gene $i$, and the matrix $B=\left(b_{i j}\right) \in \mathfrak{R}^{n \times n}$ is defined as

$$
b_{i j}:\left\{\begin{array}{cc}
>0, & \text { if transcription factor } j \\
& \text { is an activator of gene } i \\
=0, & \text { if there is no link from gene } i \text { to gene } j \\
<0, & \text { if transcription factor } j
\end{array} .\right.
$$

For simplicity, the GRN $\Sigma$ can be rewritten in the following compact matrix form:

$$
\Sigma^{\prime}:\left\{\begin{array}{l}
\frac{d m(t)}{d t}=-A m(t)+B f(p(t-\sigma))+\eta \\
\frac{d p(t)}{d t}=-C p(t)+D m(t-\tau)
\end{array}\right.
$$

where $m(t)=\operatorname{col}\left\{m_{1}(t), m_{2}(t), \ldots, m_{n}(t)\right\}, \quad p(t)=$ $\operatorname{col}\left\{p_{1}(t), p_{2}(t), \ldots, p_{n}(t)\right\}, A=\operatorname{diag}\left\{a_{1}, a_{2}, \ldots, a_{n}\right\}, C=$ $\operatorname{diag}\left\{c_{1}, c_{2}, \ldots, c_{n}\right\}, D=\operatorname{diag}\left\{d_{1}, d_{2}, \ldots, d_{n}\right\}, \eta=\operatorname{col}\left\{\eta_{1}\right.$, $\left.\eta_{2}, \ldots, \eta_{n}\right\}$, and $f(p(t-\sigma))=\operatorname{col}\left\{f_{1}\left(p_{1}(t-\sigma)\right), f_{2}\left(p_{2}(t-\right.\right.$ $\left.\sigma)), \ldots, f_{n}\left(p_{n}(t-\sigma)\right)\right\} \in \mathfrak{R}^{n}$.

The initial condition of the GRN $\Sigma^{\prime}$ is given by

$$
m(t)=\phi(t) \quad p(t)=\varphi(t) \quad-\varrho \leq t \leq 0 \quad \varrho \triangleq \max \{\tau, \sigma\}
$$

where $\phi(\cdot)$ and $\varphi(\cdot)$ are continuous functions.

Let $\operatorname{col}\left\{m^{*}, p^{*}\right\} \in \mathfrak{R}^{2 n}$ be an equilibrium point of $\Sigma^{\prime}$, which is a solution of the following nonlinear equations:

$$
\left\{\begin{array}{l}
-A m+B f(p)+\eta=0 \\
-C p+D m=0
\end{array} .\right.
$$

In the following, let us shift the unknown equilibrium point $\operatorname{col}\left\{m^{*}, p^{*}\right\}$ to the origin by defining

$$
x=m-m^{*} \quad y=p-p^{*}
$$


then, the system $\left(\Sigma^{\prime}\right)$ becomes

$$
\Sigma^{\prime \prime}:\left\{\begin{array}{l}
\frac{d x(t)}{d t}=-A x(t)+B g(y(t-\sigma)) \\
\frac{d y(t)}{d t}=-C y(t)+D x(t-\tau)
\end{array}\right.
$$

where $g(y(t))=\operatorname{col}\left\{g_{1}\left(y_{1}(t)\right), g_{2}\left(y_{2}(t)\right), \ldots, g_{n}\left(y_{n}(t)\right)\right\}$, with the $i$ th component being $g_{i}\left(y_{i}(t)\right)=f_{i}\left(y_{i}(t)+p_{i}^{*}\right)-f_{i}\left(p_{i}^{*}\right)$.

As discussed in Section I, the GRN model parameters identified from real-world time series are largely dependent on the selection of fixed points, and the relevant constants vary with the experiment data. Therefore, we further take the structure uncertainties into account and have the following more generalized model:

$\Sigma^{\prime \prime \prime}:\left\{\begin{array}{l}\frac{d x(t)}{d t}=-(A+\Delta A(t)) x(t)+(B+\Delta B(t)) g(y(t-\sigma)) \\ \frac{d y(t)}{d t}=-(C+\Delta C(t)) y(t)+(D+\Delta D(t)) x(t-\tau)\end{array}\right.$.

Here, $\Delta A(t), \Delta B(t), \Delta C(t)$, and $\Delta D(t)$ are unknown matrices with appropriate dimensions denoting the uncertain parameters, which satisfy the following admissible uncertainty condition:

$$
\left[\begin{array}{ll}
\Delta A(t) & \Delta B(t) \\
\Delta C(t) & \Delta D(t)
\end{array}\right]=\left[\begin{array}{l}
M_{1} \\
M_{2}
\end{array}\right] H(t)\left[\begin{array}{ll}
N_{1} & N_{2}
\end{array}\right]
$$

where $M_{i}$ and $N_{i}(i=1,2)$ are known real constant matrices, and $H(t)$ is a time-varying and unknown Lebesgue-measurable matrix-valued function subjected to the following condition:

$$
H^{T}(t) H(t) \leq I \quad \forall t>0 .
$$

Remark 1: In practice, this kind of norm-bounded uncertainty described by (8) and (9) is frequently encountered in many engineering problems of robust analysis of uncertain dynamic systems (see, for instance, [30] and the references therein), which may result from the variation of operating points, aging of devices, identification errors, etc. Many practical systems possess parameter uncertainties that can be either exactly modeled or over bounded by (9).

For the sake of convenience, we denote the following hereafter:

$$
\begin{array}{ll}
A(t)=A+\Delta A(t) & B(t)=B+\Delta B(t) \\
C(t)=C+\Delta C(t) & D(t)=D+\Delta D(t) .
\end{array}
$$

Assumption 1: The nonlinear function $f_{i}(\cdot)$ is continuous and bounded and satisfies the following inequality:

$$
0 \leq \frac{f_{j}(u)-f_{j}(v)}{u-v} \leq \kappa_{j}, \quad j=1,2, \ldots, n
$$

for all $u, v \in \Re, u \neq v$.

Remark 2: It follows from Assumption 1 that the nonlinear feedback regulation function $g_{j}(\cdot)$ in system $\Sigma^{\prime \prime \prime}$ satisfies the sector-like condition, i.e., $0 \leq g_{i}(s) / s \leq \kappa_{i} \forall s \neq 0$, and $g_{i}(0)=0, i=1,2, \ldots, n$, which is equivalent to $g^{T}(y)[g(y)-$ $K y] \leq 0$ with $K=\operatorname{diag}\left\{\kappa_{1}, \kappa_{2}, \ldots, \kappa_{n}\right\}$. Moreover, it should be pointed out that this sector-like condition described by (10) is more general than those that have been used in [13], [14], and [24], where the derivative for each component of the regulatory function is assumed to be the same, which is unrealistic. In our description, such a restriction is removed.

Remark 3: Usually, various fixed-point theorems, such as Brouwer's fixed-point theorem, Schauder's fixed-point theorem, and the contraction mapping principle, can be exploited to prove the existence of equilibrium points of the addressed GRNs. For example, under Assumption 1, it is not difficult to ensure the existence of an equilibrium point of the system [see (7)] by using Brouwer's fixed-point theorem. In the sequel, we shall analyze the globally asymptotic stability of the equilibrium point, which in turn implies the uniqueness of the equilibrium point.

Before stating the main results, we introduce the following useful definitions and lemmas.

Definition 1: Let the equilibrium point of the nominal system of $\Sigma^{\prime \prime}$ be stable in the sense of Lyapunov. The nominal system of $\Sigma^{\prime \prime}$ is said to be globally asymptotically stable if

$$
\lim _{t \rightarrow+\infty}\left\{\left|m(t)-m^{*}\right|^{2}+\left|p(t)-p^{*}\right|^{2}\right\}=0 .
$$

Definition 2: The uncertain system $\Sigma^{\prime \prime \prime}$ is said to be globally asymptotically robustly stable if system $\Sigma^{\prime \prime}$ is globally asymptotically stable for all admissible uncertainties.

Lemma 1 (Schur's Complement) [34]: Given any real matrices $\Omega_{1}, \Omega_{2}$, and $\Omega_{3}$, where $\Omega_{1}^{T}=\Omega_{1}$, and $\Omega_{2}>0$, then

$$
\Omega_{1}+\Omega_{3}^{T} \Omega_{2}^{-1} \Omega_{3}<0
$$

if and only if

$$
\left[\begin{array}{cc}
\Omega_{1} & \Omega_{3}^{T} \\
\Omega_{3} & -\Omega_{2}
\end{array}\right]<0
$$

Lemma 2 [31]: Assume that $D, E$ are real matrices with appropriate dimensions, and $H(t)$ is a real matrix function satisfying $H^{T}(t) H(t) \leq I$. Then, for any positive scalar $\varepsilon$, the following inequality holds:

$$
D H(t) E+(D H(t) E)^{T} \leq \varepsilon E E^{T}+\varepsilon^{-1} D^{T} D .
$$

Lemma 3 (Jensen's Inequality) [6]: Given a positive-definite matrix $P \in \mathfrak{R}^{n \times n}$ and a scalar $\pi>0$ for any vector $x(t)=$ $\operatorname{col}\left\{x_{1}(t), x_{2}(t), \ldots, x_{n}(t)\right\}$, we have

$$
\begin{aligned}
\int_{t-\pi}^{t} \dot{x}^{T}(\omega) & P \dot{x}(\omega) d \omega \\
\geq & \frac{1}{\pi}[x(t)-x(t-\pi)]^{T} P[x(t)-x(t-\pi)] .
\end{aligned}
$$

\section{Main Results}

In this section, we aim to establish the general robust stability results for the uncertain GRN with and without noise perturbations. A Lyapunov functional method is developed based on the idea of "delay fractioning" proposed in the literature. To estimate the upper bound of the time delays for stability, we partition $\tau$ and $\sigma$ into several equal components, that is, $\tau=$ $\sum_{i=1}^{r} \tau_{i}$ with $\tau_{i}=\tau / r$, and $\sigma=\sum_{i=1}^{r} \sigma_{i}$ with $\sigma_{i}=\sigma / r$, where $r$ 
is a positive integer denoting the number of fractions. Our main results are delay dependent, which are formulated in terms of LMIs to ensure the robustly asymptotic stability of the proposed uncertain GRNs with and without noise perturbations.

\section{A. Robust Stability Analysis of GRNs Without Noise Perturbations}

In this section, a theorem is presented to give the stability condition for uncertain GRNs $\Sigma / \prime \prime \prime$ without noise perturbations. Some corollaries are then obtained for the special case when there are no parameter uncertainties.

Theorem 1: Given any integer $r \geq 1$, the uncertain system $\Sigma^{\prime \prime \prime}$ is globally asymptotically robustly stable with time delays $\tau \in\left(0, h_{1}\right], \sigma \in\left(0, h_{2}\right]$ if there exist matrices $P_{i}>0, S_{i}>0$, and $\Gamma=\operatorname{diag}\left\{\gamma_{1}, \gamma_{2}, \ldots, \gamma_{n}\right\}>0$, any matrices $X_{j}^{(i)}, Y_{j}^{(i)}$, and $Z_{j}^{(i)}(i=1,2$ and $j=1, \ldots, r)$, and positive scalars $\varepsilon_{k}$ $(k=1,2, \ldots, 8)$ satisfying

$$
\left[\begin{array}{cc}
X_{j}^{(i)} & Y_{j}^{(i)} \\
* & Z_{j}^{(i)}
\end{array}\right]>0 \quad \Xi=\left[\begin{array}{cc}
\Xi_{1} & \Xi_{2} \\
\Xi_{2}^{T} & \Xi_{3}
\end{array}\right]<0
$$

where $\Xi_{1}=\Sigma_{k=1}^{2} \Xi_{1 k}, \Xi_{2}=\Omega_{1}^{T} \Phi$, and

$$
\begin{aligned}
& \Xi_{3}=-\operatorname{diag}\left\{\varepsilon_{1} I_{n}, \varepsilon_{2} I_{n}, \varepsilon_{5} I_{n}, \varepsilon_{6} I_{n}, \varepsilon_{3} I_{n},\right. \\
& \left.\varepsilon_{4} I_{n}, \varepsilon_{7} I_{n}, \varepsilon_{8} I_{n}\right\} \\
& \Xi_{11}=W_{P_{1}}^{T} \tilde{P}_{1} W_{P_{1}}+W_{P_{2}}^{T} \tilde{P}_{2} W_{P_{2}}+W_{\Gamma}^{T} \tilde{\Gamma} W_{\Gamma}+\Lambda_{P_{1}}^{T} \tilde{P}_{1} \Lambda_{P_{1}} \\
& +\Lambda_{P_{2}}^{T} \tilde{P}_{2} \Lambda_{P_{2}}+\Omega_{2}^{T} \Psi \Omega_{2} \\
& \Xi_{12}=U_{1}^{T} \mathcal{X} U_{1}-U_{2}^{T} \mathcal{X} U_{2}+V_{1}^{T} \mathcal{Y} V_{1}-V_{2}^{T} \mathcal{Y} V_{2} \\
& +Q_{1}^{T} \mathcal{S}_{1} Q_{1}+Q_{2}^{T} \mathcal{S}_{2} Q_{2} \\
& W_{P_{1}}=\left[\begin{array}{ccc}
-A & 0_{n,(4 r+3) n} & B \\
\hline I_{n} & 0_{n, 4(r+1) n}
\end{array}\right] \\
& W_{P_{2}}=\left[\begin{array}{lllll}
0_{n, r n} & D & 0_{n,(r+1) n} & -C & 0_{n, 2(r+1) n} \\
\hline 0_{n, 2(r+1) n} & I_{n} & 0_{n, 2(r+1) n}
\end{array}\right] \\
& \tilde{P}_{i}=\left[\begin{array}{cc}
0 & P_{i} \\
P_{i} & 0
\end{array}\right] \quad \tilde{\Gamma}=\left[\begin{array}{cc}
0 & \Gamma \\
\Gamma & -\Gamma
\end{array}\right] \\
& W_{\Gamma}=\left[\begin{array}{lll}
0_{n,(3 r+2) n} & \frac{\sqrt{2}}{2} K & 0_{n,(r+2) n} \\
\hline 0_{n, 4(r+1) n} & \sqrt{2} I_{n}
\end{array}\right] \\
& \Lambda_{P_{1}}=\left[\begin{array}{ccccc}
-A & 0_{n, r n} & -I_{n} & 0_{n,(3 r+2) n} & B \\
\hline 0_{n,(r+1) n} & I_{n} & 0_{n, 3(r+1) n}
\end{array}\right] \\
& \Lambda_{P_{2}}=\left[\begin{array}{llllll}
0_{n, r n} D & 0_{n,(r+1) n} & -C & 0_{n, r n} & -I_{n} & 0_{n,(r+1) n} \\
\hline 0_{n, 3(r+1) n} & I_{n} & 0_{n,(r+1) n}
\end{array}\right] \\
& U_{1}=\left[\begin{array}{ccc}
I_{r n} & 0_{r n,(3 r+5) n} \\
\hline 0_{r n,(r+1) n} & I_{r n} & 0_{r n, 2(r+2) n}
\end{array}\right] \\
& U_{2}=\left[\begin{array}{ccc}
0_{r n, n} & I_{r n} & 0_{r n,(3 r+4) n} \\
\hline 0_{r n,(r+2) n} & I_{r n} & 0_{r n,(2 r+3) n}
\end{array}\right] \\
& V_{1}=\left[\begin{array}{ccc}
0_{r n, 2(r+1) n} & I_{r n} & 0_{r n,(r+3) n} \\
\hline 0_{r n, 3(r+1) n} & I_{r n} & 0_{r n, 2 n}
\end{array}\right] \\
& V_{2}=\left[\begin{array}{ccc}
0_{r n,(2 r+3) n} & I_{r n} & 0_{r n,(r+2) n} \\
\hline 0_{r n,(3 r+4) n} & I_{r n} & 0_{r n, n}
\end{array}\right] \\
& \mathcal{X}=\left[\begin{array}{cc}
X^{(1)} & Y^{(1)} \\
* & Z^{(1)}
\end{array}\right] \quad \mathcal{Y}=\left[\begin{array}{cc}
X^{(2)} & Y^{(2)} \\
* & Z^{(2)}
\end{array}\right]
\end{aligned}
$$

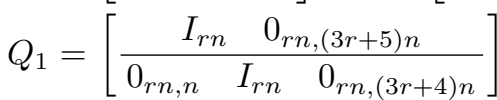

$$
\begin{aligned}
& Q_{2}=\left[\begin{array}{lll}
0_{r n,(2 r+2) n} & I_{r n} & 0_{r n,(r+3) n} \\
\hline 0_{r n,(2 r+3) n} & I_{r n} & 0_{r n,(r+2) n}
\end{array}\right] \\
& \mathcal{S}_{i}=\left[\begin{array}{cc}
S^{(i)} & -S^{(i)} \\
* & S^{(i)}
\end{array}\right]
\end{aligned}
$$

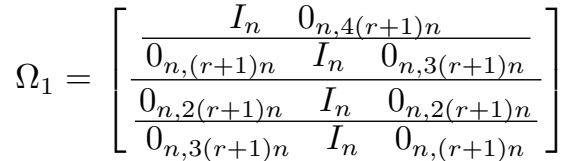

$$
\begin{aligned}
& \Omega_{2}=\left[\begin{array}{ccc}
\frac{I_{n}}{0_{n, r n}} & 0_{n, 4(r+1) n} & 0_{n,(3 r+4) n} \\
\hline 0_{n,(r+1) n} & I_{n} & 0_{n,(3 r+3) n} \\
\hline 0_{n, 2(r+1) n} & I_{n} & 0_{n,(2 r+2) n} \\
\hline 0_{n, 3(r+1) n} & I_{n} & 0_{n,(r+1) n} \\
\hline 0_{n, 4(r+1) n} & I_{n}
\end{array}\right] \\
& \Phi=\left[\begin{array}{cccc}
\frac{P_{1} M_{1}}{P_{1} M_{1}} 0_{n, 6 n} \\
\hline 0_{n, 2 n} & P_{1} M_{1} & P_{1} M_{1} & 0_{n, 4 n} \\
\hline 0_{n, 4 n} & P_{2} M_{2} & P_{2} M_{2} & 0_{n, 2 n} \\
\hline 0_{n, 6 n} & P_{2} M_{2} & P_{2} M_{2}
\end{array}\right] \\
& \Psi=\operatorname{diag}\left\{\left(\varepsilon_{1}+\varepsilon_{5}\right) N_{1}^{T} N_{1},\left(\varepsilon_{2}+\varepsilon_{4}\right) N_{2}^{T} N_{2}, h_{1} S_{1},\right. \\
& \left.\left(\varepsilon_{3}+\varepsilon_{7}\right) N_{1}^{T} N_{1}, h_{2} S_{2},\left(\varepsilon_{2}+\varepsilon_{6}\right) N_{2}^{T} N_{2}\right\} \\
& X^{(i)}=\operatorname{diag}\left\{X_{1}^{(i)}, X_{2}^{(i)}, \ldots, X_{r}^{(i)}\right\} \\
& Y^{(i)}=\operatorname{diag}\left\{Y_{1}^{(i)}, Y_{2}^{(i)}, \ldots, Y_{r}^{(i)}\right\} \\
& Z^{(i)}=\operatorname{diag}\left\{Z_{1}^{(i)}, Z_{2}^{(i)}, \ldots, Z_{r}^{(i)}\right\} \\
& S^{(i)}=-\frac{r}{h_{i}}\left(I_{r} \otimes S_{i}\right), \quad i=1,2 .
\end{aligned}
$$

Proof: Based on Lemma 1, since $\Xi_{3}<0$, (12) is equivalent to $\Xi_{1}-\Xi_{2} \Xi_{3}^{-1} \Xi_{2}^{T}<0$. To prove the theorem, we choose a novel Lyapunov-Krasovskii functional candidate as follows:

$$
V(t)=V_{1}(t)+V_{2}(t)+V_{3}(t)
$$

where

$$
\begin{aligned}
V_{1}(t)= & x^{T}(t) P_{1} x(t) \\
& +\sum_{k=1}^{r} \int_{t-\frac{k}{r} \tau}^{t-\frac{k-1}{r} \tau}\left[\begin{array}{l}
x(s) \\
\dot{x}(s)
\end{array}\right]^{T}\left[\begin{array}{cc}
X_{k}^{(1)} & Y_{k}^{(1)} \\
* & Z_{k}^{(1)}
\end{array}\right]\left[\begin{array}{l}
x(s) \\
\dot{x}(s)
\end{array}\right] d s \\
V_{2}(t)= & y^{T}(t) P_{2} y(t) \\
& +\sum_{k=1}^{r} \int_{t-\frac{k}{r} \sigma}^{t-\frac{k-1}{r} \sigma}\left[\begin{array}{l}
y(s) \\
\dot{y}(s)
\end{array}\right]^{T}\left[\begin{array}{cc}
X_{k}^{(2)} & Y_{k}^{(2)} \\
* & Z_{k}^{(2)}
\end{array}\right]\left[\begin{array}{l}
y(s) \\
\dot{y}(s)
\end{array}\right] d s \\
V_{3}(t)= & \int_{-\tau}^{0} \int_{t+\beta}^{t} \dot{x}^{T}(\alpha) S_{1} \dot{x}(\alpha) d \alpha d \beta \\
& +\int_{-\sigma}^{0} \int_{t+\nu}^{t} \dot{y}^{T}(\mu) S_{2} \dot{y}(\mu) d \mu d \nu
\end{aligned}
$$

with $r \geq 1$ (number of fractions) being an integer. 
Considering the derivatives of $V_{i}(t)(i=1,2,3)$ along the trajectory of system $\Sigma^{\prime \prime \prime}$, we have

$$
\begin{aligned}
\dot{V}_{1}(t)= & 2 x^{T}(t) P_{1} \dot{x}(t)+\left[\begin{array}{c}
x(t) \\
\dot{x}(t)
\end{array}\right]^{T}\left[\begin{array}{cc}
X_{1}^{(1)} & Y_{1}^{(1)} \\
* & Z_{1}^{(1)}
\end{array}\right]\left[\begin{array}{l}
x(t) \\
\dot{x}(t)
\end{array}\right] \\
& -\left[\begin{array}{c}
x(t-\tau) \\
\dot{x}(t-\tau)
\end{array}\right]^{T}\left[\begin{array}{cc}
X_{r}^{(1)} & Y_{r}^{(1)} \\
* & Z_{r}^{(1)}
\end{array}\right]\left[\begin{array}{l}
x(t-\tau) \\
\dot{x}(t-\tau)
\end{array}\right] \\
& -\sum_{l=1}^{r-1}\left(\left[\begin{array}{c}
x\left(t-\frac{l}{r} \tau\right) \\
\dot{x}\left(t-\frac{l}{r} \tau\right)
\end{array}\right]^{T}\left[\begin{array}{cc}
X_{l}^{(1)}-X_{l+1}^{(1)} & Y_{l}^{(1)}-Y_{l+1}^{(1)} \\
* & Z_{l}^{(1)}-Z_{l+1}^{(1)}
\end{array}\right]\right. \\
& \left.\times\left[\begin{array}{c}
x\left(t-\frac{l}{r} \tau\right) \\
\dot{x}\left(t-\frac{l}{r} \tau\right)
\end{array}\right]\right) \\
= & 2 x^{T}(t) P_{1}[-A(t) x(t)+B(t) g(y(t-\sigma))]+\left[\begin{array}{l}
\mathcal{X}(t) \\
\dot{\mathcal{X}}(t)
\end{array}\right]^{T} \\
& \times\left[\begin{array}{cc}
X^{(1)} & Y^{(1)} \\
* & Z^{(1)}
\end{array}\right]\left[\begin{array}{l}
\mathcal{X}(t) \\
\dot{\mathcal{X}}(t)
\end{array}\right]-\left[\begin{array}{l}
\mathcal{X}\left(t-\frac{\tau}{r}\right) \\
\dot{\mathcal{X}}\left(t-\frac{\tau}{r}\right)
\end{array}\right]^{T} \\
& \times\left[\begin{array}{cc}
X^{(1)} & Y^{(1)} \\
* & Z^{(1)}
\end{array}\right]\left[\begin{array}{l}
\mathcal{X}\left(t-\frac{\tau}{r}\right) \\
\dot{\mathcal{X}}\left(t-\frac{\tau}{r}\right)
\end{array}\right]
\end{aligned}
$$$$
\dot{V}_{2}(t)=2 y^{T}(t) P_{2} \dot{y}(t)+\left[\begin{array}{c}
y(t) \\
\dot{y}(t)
\end{array}\right]^{T}\left[\begin{array}{cc}
X_{1}^{(2)} & Y_{1}^{(2)} \\
* & Z_{1}^{(2)}
\end{array}\right]\left[\begin{array}{c}
y(t) \\
\dot{y}(t)
\end{array}\right]
$$$$
-\left[\begin{array}{c}
y(t-\sigma) \\
\dot{y}(t-\sigma)
\end{array}\right]^{T}\left[\begin{array}{cc}
X_{r}^{(2)} & Y_{r}^{(2)} \\
* & Z_{r}^{(2)}
\end{array}\right]\left[\begin{array}{c}
y(t-\sigma) \\
\dot{y}(t-\sigma)
\end{array}\right]
$$$$
-\sum_{l=1}^{r-1}\left(\left[\begin{array}{c}
y\left(t-\frac{l}{r} \sigma\right) \\
\dot{y}\left(t-\frac{l}{r} \sigma\right)
\end{array}\right]^{T}\left[\begin{array}{cc}
X_{l}^{(2)}-X_{l+1}^{(2)} & Y_{l}^{(2)}-Y_{l+1}^{(2)} \\
* & Z_{l}^{(2)}-Z_{l+1}^{(2)}
\end{array}\right]\right.
$$$$
\left.\times\left[\begin{array}{c}
y\left(t-\frac{l}{r} \sigma\right) \\
\dot{y}\left(t-\frac{l}{r} \sigma\right)
\end{array}\right]\right)
$$$$
=2 y^{T}(t) P_{2}[-C(t) y(t)+D(t) x(t-\tau)]+\left[\begin{array}{c}
\mathcal{Y}(t) \\
\dot{\mathcal{Y}}(t)
\end{array}\right]^{T}
$$$$
\times\left[\begin{array}{cc}
X^{(2)} & Y^{(2)} \\
* & Z^{(2)}
\end{array}\right]\left[\begin{array}{l}
\mathcal{Y}(t) \\
\dot{\mathcal{Y}}(t)
\end{array}\right]-\left[\begin{array}{c}
\mathcal{Y}\left(t-\frac{\sigma}{r}\right) \\
\dot{\mathcal{Y}}\left(t-\frac{\sigma}{r}\right)
\end{array}\right]^{T}
$$$$
\times\left[\begin{array}{cc}
X^{(2)} & Y^{(2)} \\
* & Z^{(2)}
\end{array}\right]\left[\begin{array}{l}
\mathcal{Y}\left(t-\frac{\sigma}{r}\right) \\
\dot{\mathcal{Y}}\left(t-\frac{\sigma}{r}\right)
\end{array}\right]
$$$$
\dot{V}_{3}(t)=\tau \dot{x}^{T}(t) S_{1} \dot{x}(t)+\sigma \dot{y}^{T}(t) S_{2} \dot{y}(t)
$$$$
-\int_{t-\tau}^{t} \dot{x}^{T}(\alpha) S_{1} \dot{x}(\alpha) d \alpha
$$$$
-\int_{t-\sigma}^{t} \dot{y}^{T}(\mu) S_{2} \dot{y}(\mu) d \mu
$$$$
\leq h_{1} \dot{x}^{T}(t) S_{1} \dot{x}(t)+h_{2} \dot{y}^{T}(t) S_{2} \dot{y}(t)
$$$$
-\sum_{k=1}^{r} \int_{t-\frac{k}{r} \tau}^{t-\frac{k-1}{r} \tau} \dot{x}^{T}(\alpha) S_{1} \dot{x}(\alpha) d \alpha
$$$$
-\sum_{k=1}^{r} \int_{t-\frac{k}{r} \sigma}^{t-\frac{k-1}{r} \sigma} \dot{y}^{T}(\mu) S_{2} \dot{y}(\mu) d \mu
$$

where $\mathcal{X}(t)=\operatorname{col}\{x(t), x(t-(1 / r) \tau), \ldots, x(t-((r-1) / r) \tau)\}$, and $\mathcal{Y}(t)=\operatorname{col}\{y(t), y(t-(1 / r) \sigma), \ldots, y(t-((r-1) / r) \sigma)\}$.
From Lemma 3, for $k=1,2, \ldots, r$, it readily follows that

$$
\begin{aligned}
& -\int_{t-\frac{k}{r} \tau}^{t-\frac{k-1}{r} \tau} \dot{x}^{T}(\alpha) S_{1} \dot{x}(\alpha) d \alpha \\
\leq & -\frac{r}{h_{1}}\left[x\left(t-\frac{k-1}{r} \tau\right)-x\left(t-\frac{k}{r} \tau\right)\right]^{T} S_{1} \\
\times & {\left[x\left(t-\frac{k-1}{r} \tau\right)-x\left(t-\frac{k}{r} \tau\right)\right] ; } \\
& -\int_{t-\frac{k-1}{r} \sigma} \sigma \\
\leq & -\frac{r}{h_{2}}\left[y\left(t-\frac{k-1}{r} \sigma\right)-y\left(t-\frac{k}{r} \sigma\right)\right]^{T} S_{2} \dot{y}(\mu) d \mu \\
\times & {\left[y\left(t-\frac{k-1}{r} \sigma\right)-y\left(t-\frac{k}{r} \sigma\right)\right] }
\end{aligned}
$$

and then

$$
\begin{aligned}
& -\sum_{k=1}^{r} \int_{t-\frac{k}{r} \tau}^{t-\frac{k-1}{r} \tau} \dot{x}^{T}(\alpha) S_{1} \dot{x}(\alpha) d \alpha \\
\leq & \mathcal{X}^{T}(t) S^{(1)} \mathcal{X}(t)+\mathcal{X}^{T}\left(t-\frac{\tau}{r}\right) S^{(1)} \mathcal{X}\left(t-\frac{\tau}{r}\right) \\
& -2 \mathcal{X}^{T}(t) S^{(1)} \mathcal{X}\left(t-\frac{\tau}{r}\right) \\
& -\sum_{k=1}^{r} \int_{t-\frac{k}{r} \sigma}^{t-\frac{k-1}{r} \sigma} \dot{y}^{T}(\mu) S_{2} \dot{y}(\mu) d \mu \\
\leq & \mathcal{Y}^{T}(t) S^{(2)} \mathcal{Y}(t)+\mathcal{Y}^{T}\left(t-\frac{\sigma}{r}\right) S^{(2)} \mathcal{Y}\left(t-\frac{\sigma}{r}\right) \\
& -2 \mathcal{Y}^{T}(t) S^{(2)} \mathcal{Y}\left(t-\frac{\sigma}{r}\right)
\end{aligned}
$$

where $S^{(i)}=-\left(r / h_{i}\right)\left(I_{r} \otimes S_{i}\right), i=1,2$.

From the sector condition in Assumption 1, for any scalar $\gamma_{j}>0$, one can see that

$$
-2 \sum_{j=1}^{n} \gamma_{j} g_{j}\left(y_{j}(t-\sigma)\right)\left[g_{j}\left(y_{j}(t-\sigma)\right)-\kappa_{i} y_{j}(t-\sigma)\right] \geq 0
$$

which is equivalent to

$$
\begin{aligned}
& 2 g^{T}(y(t-\sigma)) \Gamma K y(t-\sigma) \\
& \quad-2 g^{T}(y(t-\sigma)) \Gamma g(y(t-\sigma)) \leq 0
\end{aligned}
$$

where $\Gamma=\operatorname{diag}\left\{\gamma_{1}, \gamma_{2}, \ldots, \gamma_{n}\right\}$.

In view of (7), we obtain

$$
\begin{aligned}
\dot{x}^{T}(t) P_{1}[-A(t) x(t)+B(t) g(y(t-\sigma))-\dot{x}(t)] & =0 \\
\dot{y}^{T}(t) P_{2}[-C(t) y(t)+D(t) x(t-\tau)-\dot{y}(t)] & =0 .
\end{aligned}
$$


Using (8), (9), and Lemma 2, for positive scalars $\varepsilon_{i}>0(i=$ $1,2, \ldots, 8)$, we have

$$
\begin{aligned}
&- 2 x^{T}(t) P_{1} \Delta A(t) x(t) \\
& \leq x^{T}(t)\left[\varepsilon_{1}^{-1} P_{1} M_{1} M_{1}^{T} P_{1}+\varepsilon_{1} N_{1}^{T} N_{1}\right] x(t) \\
& 2 x^{T}(t) P_{1} \Delta B(t) g(y(t-\sigma)) \\
& \leq \varepsilon_{2}^{-1} x^{T}(t) P_{1} M_{1} M_{1}^{T} P_{1} x(t)+\varepsilon_{2} g^{T}(y(t-\sigma)) \\
& \times N_{2}^{T} N_{2} g(y(t-\sigma)) \\
&- 2 y^{T}(t) P_{2} \Delta C(t) y(t) \\
& \leq y^{T}(t)\left[\varepsilon_{3}^{-1} P_{2} M_{2} M_{2}^{T} P_{2}+\varepsilon_{3} N_{1}^{T} N_{1}\right] y(t) \\
& 2 y^{T}(t) P_{2} \Delta D(t) x(t-\tau) \\
& \leq \\
& \quad \varepsilon_{4}^{-1} y^{T}(t) P_{2} M_{2} M_{2}^{T} P_{2} y(t)+\varepsilon_{4} x^{T}(t-\tau) \\
& \quad \times N_{2}^{T} N_{2} x(t-\tau)
\end{aligned}
$$

and similarly

$$
\begin{aligned}
-2 \dot{x}^{T}(t) P_{1} \Delta A(t) x(t) \leq & \varepsilon_{5}^{-1} \dot{x}^{T}(t) P_{1} M_{1} M_{1}^{T} P_{1} \dot{x}(t) \\
& +\varepsilon_{5} x^{T}(t) N_{1}^{T} N_{1} x(t) \\
2 \dot{x}^{T}(t) P_{1} \Delta B(t) g(y(t-\sigma)) \leq & \varepsilon_{6}^{-1} \dot{x}^{T}(t) P_{1} M_{1} M_{1}^{T} P_{1} \dot{x}(t) \\
& +\varepsilon_{6} g^{T}(y(t-\sigma)) \\
& \times N_{2}^{T} N_{2} g(y(t-\sigma)) \\
-2 \dot{y}^{T}(t) P_{2} \Delta C(t) y(t) \leq & \varepsilon_{7}^{-1} \dot{y}^{T}(t) P_{2} M_{2} M_{2}^{T} P_{2} \dot{y}(t) \\
& +\varepsilon_{7} y^{T}(t) N_{1}^{T} N_{1} y(t) \\
2 \dot{y}^{T}(t) P_{2} \Delta D(t) x(t-\tau) \leq & \varepsilon_{8}^{-1} \dot{y}^{T}(t) P_{2} M_{2} M_{2}^{T} P_{2} \dot{y}(t) \\
& +\varepsilon_{8} x^{T}(t-\tau) \\
& \times N_{2}^{T} N_{2} x(t-\tau) .
\end{aligned}
$$

Now, it follows from (14)-(29) and Lemma 1 that

$$
\dot{V}(t) \leq \xi^{T}(t)\left(\Xi_{1}-\Xi_{2} \Xi_{3}^{-1} \Xi_{2}^{T}\right) \xi(t)
$$

where $\xi(t)=\operatorname{col}\{\mathcal{X}(t), x(t-\tau), \dot{\mathcal{X}}(t), \dot{x}(t-\tau), \mathcal{Y}(t), y(t-$ $\sigma), \dot{\mathcal{Y}}(t), \dot{y}(t-\sigma), g(y(t-\sigma))\}$, and $\Xi_{i}(i=1,2,3)$ is defined in (12).

Furthermore, the condition in (12) indicates that there exists a positive scalar $\lambda$ such that

$$
\dot{V}(t) \leq-\lambda\left(|x(t)|^{2}+|y(t)|^{2}\right)
$$

which implies from the Lyapunov stability theory that the GRN in (7) is robustly globally asymptotically stable. Hence, the proof is completed.

For the nominal system $\Sigma^{\prime \prime}$ without parameter uncertainties, according to Theorem 1, it is not difficult to establish the following sufficient condition on the globally asymptotic stability.

Corollary 1: Given any integer $r \geq 1$, the nominal system of genetic networks $\Sigma$ III with time delays $\tau \in\left(0, h_{1}\right]$ and $\sigma \in\left(0, h_{2}\right]$ is globally asymptotically stable if there exist matrices $P_{i}>0, S_{i}>0, \Gamma=\operatorname{diag}\left\{\gamma_{1}, \gamma_{2}, \ldots, \gamma_{n}\right\}>0$, and $\left[\begin{array}{cc}X_{j}^{(i)} & Y_{j}^{(i)} \\ * & Z_{j}^{(i)}\end{array}\right]>0(i=1,2$ and $j=1, \ldots, r)$ satisfying

$$
\tilde{\Xi}_{1}=\tilde{\Xi}_{11}+\Xi_{12}<0
$$

where

$$
\begin{aligned}
\tilde{\Xi}_{11}= & W_{P_{1}}^{T} \tilde{P}_{1} W_{P_{1}}+W_{P_{2}}^{T} \tilde{P}_{2} W_{P_{2}}+W_{\Gamma}^{T} \tilde{\Gamma} W_{\Gamma} \\
& +\Lambda_{P_{1}}^{T} \tilde{P}_{1} \Lambda_{P_{1}}+\Lambda_{P_{2}}^{T} \tilde{P}_{2} \Lambda_{P_{2}}+\tilde{\Omega}_{2}^{T} \tilde{\Psi} \tilde{\Omega}_{2} \\
\tilde{\Omega}_{2}= & {\left[\begin{array}{lll}
0_{n,(r+1) n} & I_{n} & 0_{n, 3(r+1) n} \\
\hline 0_{n, 3(r+1) n} & I_{n} & 0_{n,(r+1) n}
\end{array}\right] } \\
\tilde{\Psi}= & \operatorname{diag}\left\{h_{1} S_{1}, h_{2} S_{2}\right\}
\end{aligned}
$$

and the other symbols have the same meaning as those defined in Theorem 1.

\section{B. Robust Stability Analysis of GRNs With Noise Perturbations}

It is now well known that the intracellular and extracellular noise perturbations are unavoidable during the modeling of genetic network models. Therefore, it would be interesting to consider the dynamics for the genetic networks with both parameter fluctuations and stochastic disturbances, and stability analysis is obviously one of the most important problems. In this section, by means of stochastic analysis theory, the globally robustly asymptotic stability conditions in the mean-square sense are obtained for the addressed uncertain stochastic GRNs.

Let us consider the GRNs with both parameter uncertainties and noise perturbations described by the following stochastic differential equations:

$$
\left\{\begin{array}{c}
d x(t)=[-A(t) x(t)+B(t) g(y(t-\sigma))] d t \\
+\rho(t, x(t), y(t-\sigma)) d \omega_{1}(t) \\
d y(t)=\left[\begin{array}{c}
-C(t) y(t)+D(t) x(t-\tau)] d t \\
+\rho(t, y(t), x(t-\tau)) d \omega_{2}(t)
\end{array}\right.
\end{array}\right.
$$

where $\omega_{1}(t)$ and $\omega_{2}(t)$ are mutually uncorrelated one dimensional Brownian motions satisfying $\mathbb{E}\left\{d \omega_{i}(t)\right\}=0$ and $\mathbb{E}\left\{d \omega_{i}^{2}(t)\right\}=d t(i=1,2)$. Furthermore, $\rho(t, x(t), y(t-\sigma))$ and $\rho(t, y(t), x(t-\tau))$ are the noise intensity functions.

Assumption 2: There exist matrices $\mathbb{U} \geq 0$ and $\mathbb{V} \geq 0$ such that

$$
\rho^{T}(t, u, v) \rho(t, u, v) \leq u^{T} \mathbb{U} u+v^{T} \mathbb{V} v
$$

holds for all $u, v \in \mathfrak{R}^{n}, t>0$.

The initial condition associated with the networks in (32) is given as follows:

$$
x(t)=\phi_{\omega}(t) \quad y(t)=\varphi_{\omega}(t) \quad-\varrho \leq t \leq 0
$$

where $\varrho \triangleq \max \{\tau, \sigma\} ; \quad \phi_{\omega}(t), \quad \varphi_{\omega}(t) \in L_{\mathcal{F}}^{2}\left([-\varrho, 0], \mathfrak{R}^{n}\right)$, and $L_{\mathcal{F}}^{2}\left([-\varrho, 0], \Re^{n}\right)$ denotes the family of all $\mathcal{F}_{0^{-}}$ measurable $\mathcal{C}\left([-\varrho, 0], \mathfrak{R}^{n}\right)$-valued random variables satisfying $\sup _{s \in[-\varrho, 0]} \mathbb{E}\left\{\left\|\phi_{\omega}(s)\right\|^{2}\right\}<\infty, \sup _{s \in[-\varrho, 0]} \mathbb{E}\left\{\left\|\varphi_{\omega}(s)\right\|^{2}\right\}<\infty$.

We are now in a position to analyze the problem of globally robust stability in the mean square sense for uncertain stochastic GRNs [see (32)] by using the theory of stochastic functional differential equations. We aim to establish criteria that ensure the solvability of the robust mean-square stability problem.

Theorem 2: Under Assumptions 1 and 2, for a given an integer $r \geq 1$, the genetic network in (32) with time delays 
$\tau \in\left(0, h_{1}\right]$ and $\sigma \in\left(0, h_{2}\right]$ is robustly asymptotically meansquare stable if there exist positive definite matrices $P_{i}, R_{i}, S_{i}$, $Q_{\alpha}^{(\beta)}$, and $\Gamma=\operatorname{diag}\left\{\gamma_{1}, \gamma_{2}, \ldots, \gamma_{n}\right\}$, matrices $\mathcal{M}_{\alpha}, \mathcal{N}_{\alpha}$, and $\mathcal{T}_{i}$, and positive constants $\ell_{i}$ and $\varepsilon_{j}(i=1,2 ; \alpha=1,2, \ldots, r$; $\beta=1,2,3 ; j=1,2, \ldots, 8)$ such that the following LMIs hold:

$$
\begin{aligned}
P_{i}+h_{i} R_{i} \leq \ell_{i} I \quad(i=1,2) \\
\Xi=\left[\begin{array}{ccc}
\Xi_{1} & \Xi_{2} & \Xi_{3} \\
* & \Xi_{4} & 0 \\
* & * & \Xi_{5}
\end{array}\right]<0
\end{aligned}
$$

where

$$
\begin{aligned}
& \Xi_{1}=\mathcal{Q}_{j}^{T} \Lambda_{j} \mathcal{Q}_{j} \quad(j=1,2,3,4) \\
& \Xi_{2}=\mathcal{W}^{T} \Omega \\
& \Xi_{3}=\Theta^{T} \Phi \\
& \Xi_{4}=\operatorname{diag}\left\{-\frac{r}{h_{1}} I_{r} \otimes S_{1},-\frac{r}{h_{2}} I_{r} \otimes S_{2},-I_{r} \otimes R_{1},\right. \\
& \left.-I_{r} \otimes R_{2}\right\} \\
& \Xi_{5}=\operatorname{diag}\left\{-\varepsilon_{5} I_{n},-\varepsilon_{6} I_{n},-\varepsilon_{1} I_{n},-\varepsilon_{2} I_{n},-\varepsilon_{7} I_{n},\right. \\
& \left.-\varepsilon_{8} I_{n},-\varepsilon_{3} I_{n},-\varepsilon_{4} I_{n}\right\}
\end{aligned}
$$

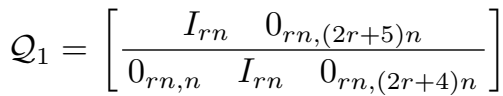

$$
\begin{aligned}
& \mathcal{Q}_{2}=\left[\begin{array}{lll}
0_{r n,(r+2) n} & I_{r n} & 0_{r n,(r+3) n} \\
\hline 0_{r n,(r+3) n} & I_{r n} & 0_{r n,(r+2) n}
\end{array}\right] \\
& \mathcal{Q}_{3}=\left[\begin{array}{ccc}
0_{r n,(r+2) n} & I_{r n} & 0_{r n,(r+3) n} \\
\hline 0_{r n,(2 r+4) n} & I_{r n} & 0_{r n, n} \\
\hline 0_{r n,(2 r+5) n} & I_{r n}
\end{array}\right] \\
& \Lambda_{1}=\left[\begin{array}{cc}
Q_{1}+\mathcal{M}+\mathcal{M}^{T} & -\mathcal{M} \\
* & -Q_{1}
\end{array}\right] \\
& \Lambda_{2}=\left[\begin{array}{cc}
Q_{2}+\mathcal{N}+\mathcal{N}^{T} & -\mathcal{N} \\
* & -Q_{2}
\end{array}\right] \\
& \Lambda_{3}=\left[\begin{array}{ccc}
0 & I_{r} \otimes K^{T} \Gamma^{T} & 0 \\
* & Q_{3}-I_{r} \otimes\left(\Gamma+\Gamma^{T}\right) & 0 \\
* & * & -Q_{3}
\end{array}\right] \\
& \mathcal{Q}_{4}=\left[\begin{array}{ccc}
\frac{I_{n}}{3} 0_{n,(3 r+4) n} \\
\hline 0_{n, r n} & I_{n} & 0_{n,(2 r+4) n} \\
\hline 0_{n,(r+1) n} & I_{n} & 0_{n,(2 r+3) n} \\
\hline 0_{n,(r+2) n} & I_{n} & 0_{n,(2 r+2) n} \\
\hline 0_{n, 2(r+1) n} & I_{n} & 0_{n,(r+2) n} \\
\hline 0_{n,(2 r+3) n} & I_{n} & 0_{n,(r+1) n} \\
\hline 0_{n,(2 r+4) n} & I_{n} & 0_{n, r n} \\
\hline 0_{n,(3 r+4) n} & I_{n}
\end{array}\right]
\end{aligned}
$$$$
\mathcal{W}=\left[\begin{array}{ccc}
\frac{I_{r n}}{0_{r n,(2 r+5) n}} \\
\hline 0_{r n,(r+2) n} & I_{r n} & 0_{r n,(r+3) n} \\
\hline I_{r n} & 0_{r n,(2 r+5) n} \\
\hline 0_{r n,(r+2) n} & I_{r n} & 0_{r n,(r+3) n}
\end{array}\right]
$$$$
\Theta=\left[\begin{array}{ccc}
I_{n} & 0_{n,(3 r+4) n} \\
\hline 0_{n,(r+1) n} & I_{n} & 0_{n,(2 r+3) n} \\
\hline 0_{n,(r+2) n} & I_{n} & 0_{n,(2 r+2) n} \\
\hline 0_{n,(2 r+3) n} & I_{n} & 0_{n,(r+1) n}
\end{array}\right]
$$$$
\Phi=\left[\begin{array}{cccc}
\frac{P_{1} M_{1}}{2} P_{1} M_{1} & 0_{n, 6 n} \\
\hline 0_{n, 2 n} & \mathcal{T}_{1} M_{1} & \mathcal{T}_{1} M_{1} & 0_{n, 4 n} \\
\hline 0_{n, 4 n} & P_{2} M_{2} & P_{2} M_{2} & 0_{n, 2 n} \\
\hline 0_{n, 6 n} & \mathcal{T}_{2} M_{2} & \mathcal{T}_{2} M_{2}
\end{array}\right]
$$$$
\Omega=\left[\begin{array}{cccc}
\mathcal{M} & 0 & \mathcal{M} & 0 \\
0 & \mathcal{N} & 0 & \mathcal{N} \\
0 & 0 & 0 & 0 \\
0 & 0 & 0 & 0
\end{array}\right]
$$$$
\Lambda_{4}=\left[\begin{array}{cc}
\Pi^{(1)} & \Pi^{(2)} \\
* & \Pi^{(3)}
\end{array}\right]
$$$$
\Pi^{(1)}=\left[\begin{array}{cccc}
\Pi_{11}^{(1)} & 0 & -A^{T} \mathcal{T}_{1}^{T} & 0 \\
0 & \Pi_{22}^{(1)} & 0 & D P_{2}^{T} \\
-\mathcal{T}_{1} A & 0 & \Pi_{33}^{(1)} & 0 \\
0 & P_{2} D & 0 & \Pi_{44}^{(1)}
\end{array}\right]
$$$$
\Pi^{(2)}=\left[\begin{array}{cccc}
0 & 0 & 0 & P_{1} B \\
0 & D^{T} \mathcal{T}_{2}^{T} & 0 & 0 \\
0 & 0 & 0 & \mathcal{T}_{1} B \\
0 & -C^{T} \mathcal{T}_{2}^{T} & 0 & 0
\end{array}\right]
$$$$
\Pi^{(3)}=\operatorname{diag}\left\{\ell_{1} \mathbb{V}, h_{2} S_{2}-\mathcal{T}_{2}-\mathcal{T}_{2}^{T}, 0,\left(\varepsilon_{2}+\varepsilon_{6}\right) N_{2}^{T} N_{2}\right\}
$$$$
\Pi_{11}^{(1)}=-P_{1} A-A^{T} P_{1}+\ell_{1} \mathbb{U}+\left(\varepsilon_{1}+\varepsilon_{5}\right) N_{1}^{T} N_{1}
$$$$
\Pi_{22}^{(1)}=\ell_{2} \mathbb{V}+\left(\varepsilon_{4}+\varepsilon_{8}\right) N_{2}^{T} N_{2}
$$$$
\Pi_{33}^{(1)}=h_{1} S_{1}-\mathcal{T}_{1}-\mathcal{T}_{1}^{T}
$$$$
\Pi_{44}^{(1)}=\ell_{2} \mathbb{U}+\left(\varepsilon_{3}+\varepsilon_{7}\right) N_{1}^{T} N_{1}-P_{2} C-C^{T} P_{2}
$$$$
\mathcal{M}=\operatorname{diag}\left\{\mathcal{M}_{1}, \mathcal{M}_{2}, \ldots, \mathcal{M}_{r}\right\}
$$$$
\mathcal{N}=\operatorname{diag}\left\{\mathcal{N}_{1}, \mathcal{N}_{2}, \ldots, \mathcal{N}_{r}\right\},
$$$$
Q_{\beta}=\operatorname{diag}\left\{Q_{1}^{(\beta)}, Q_{2}^{(\beta)}, \ldots, Q_{r}^{(\beta)}\right\} \quad(\beta=1,2,3) .
$$

\section{Proof: By setting}

$$
\begin{aligned}
& \mathfrak{x}(\alpha)=-A(\alpha) x(\alpha)+B(\alpha) g(y(\alpha-\sigma)) \\
& \mathfrak{y}(\alpha)=-C(\alpha) y(\alpha)+D(\alpha) x(\alpha-\tau)
\end{aligned}
$$

we consider the following Lyapunov-Krasovskii functional candidate for the model in (32):

$$
V(t, x(t), y(t))=\sum_{i=1}^{5} V_{i}(t, x(t), y(t))
$$


where

$$
\begin{aligned}
& V_{1}(t, x(t), y(t))=x^{T}(t) P_{1} x(t) \\
& +\sum_{k=1}^{r} \int_{t-\frac{k}{r} \tau}^{t-\frac{k-1}{r} \tau} x^{T}(s) Q_{k}^{(1)} x(s) d s \\
& V_{2}(t, x(t), y(t))=y^{T}(t) P_{2} y(t) \\
& +\sum_{k=1}^{r} \int_{t-\frac{k}{r} \sigma}^{t-\frac{k-1}{r} \sigma} y^{T}(s) Q_{k}^{(2)} y(s) d s \\
& V_{3}(t, x(t), y(t))=\sum_{k=1}^{r} \int_{t-\frac{k}{r} \sigma}^{t-\frac{k-1}{r} \sigma} g^{T}(y(s)) Q_{k}^{(3)} g(y(s)) d s \\
& V_{4}(t, x(t), y(t))=\int_{-\tau}^{0} \int_{t+\beta}^{t} \mathfrak{x}^{T}(\alpha) S_{1} \mathfrak{x}(\alpha) d \alpha d \beta \\
& +\int_{-\tau}^{0} \int_{t+\theta}^{t} \rho^{T}(s, x(s), y(s-\sigma)) \\
& \times R_{1} \rho(s, x(s), y(s-\sigma)) d s d \theta \\
& V_{5}(t, x(t), y(t))=\int_{-\sigma}^{0} \int_{t+\beta}^{t} \mathfrak{y}^{T}(\alpha) S_{2} \mathfrak{y}(\alpha) d \alpha d \beta \\
& +\int_{-\sigma}^{0} \int_{t+\theta}^{t} \rho^{T}(s, y(s), x(s-\tau)) \\
& \times R_{2} \rho(s, y(s), x(s-\tau)) d s d \theta
\end{aligned}
$$

with $r \geq 1$ (number of fractions) being an integer.

Let $\mathcal{L}$ be the weak infinitesimal operator of the stochastic process $\left\{x_{\varrho}=x(t+s), y_{\varrho}=y(t+s) \mid t \geq 0, s \in[-\varrho, 0]\right\}$ along the trajectories of the genetic network in (32). By Itô's differential formula [11], one has

$$
\begin{aligned}
\mathcal{L} V_{1}(t)= & 2 x^{T}(t) P_{1}[-A(t) x(t)+B(t) g(y(t-\sigma))] \\
& +\rho^{T}(t, x(t), y(t-\sigma)) P_{1} \rho(t, x(t), y(t-\sigma)) \\
& -\mathcal{X}^{T}\left(t-\frac{\tau}{r}\right) Q_{1} \mathcal{X}\left(t-\frac{\tau}{r}\right)+\mathcal{X}^{T}(t) Q_{1} \mathcal{X}(t)
\end{aligned}
$$

$$
\begin{aligned}
\mathcal{L} V_{2}(t)= & 2 y^{T}(t) P_{2}[-C(t) y(t)+D(t) x(t-\tau)] \\
& +\rho^{T}(t, y(t), x(t-\tau)) P_{2} \rho(t, y(t), x(t-\tau)) \\
& -\mathcal{Y}^{T}\left(t-\frac{\sigma}{r}\right) Q_{2} \mathcal{Y}\left(t-\frac{\sigma}{r}\right)+\mathcal{Y}^{T}(t) Q_{2} \mathcal{Y}(t)
\end{aligned}
$$

$$
\begin{aligned}
\mathcal{L} V_{3}(t)= & \mathcal{G}^{T}(y(t)) Q_{3} \mathcal{G}(y(t)) \\
& -\mathcal{G}^{T}\left(y\left(t-\frac{1}{r} \sigma\right)\right) Q_{3} \mathcal{G}\left(y\left(t-\frac{1}{r} \sigma\right)\right)
\end{aligned}
$$

$$
\begin{aligned}
\mathcal{L} V_{4}(t)= & \tau \mathfrak{x}^{T}(t) S_{1} \mathfrak{x}(t)-\int_{t-\tau}^{t} \mathfrak{x}^{T}(\alpha) S_{1} \mathfrak{x}(\alpha) d \alpha \\
& +\tau \rho^{T}(t, x(t), y(t-\sigma)) R_{1} \rho(t, x(t), y(t-\sigma)) \\
& -\int_{t-\tau}^{t} \rho^{T}(s, x(s), y(s-\sigma)) \\
& \times R_{1} \rho(s, x(s), y(s-\sigma)) d s \\
\mathcal{L} V_{5}(t)= & \sigma \mathfrak{y}^{T}(t) S_{2} \mathfrak{y}(t)-\int_{t-\sigma}^{t} \mathfrak{y}^{T}(\alpha) S_{2} \mathfrak{y}(\alpha) d \alpha \\
& +\sigma \rho^{T}(t, y(t), x(t-\tau)) R_{2} \rho(t, y(t), x(t-\tau)) \\
& -\int_{t-\sigma}^{t} \rho^{T}(s, y(s), x(s-\tau)) \\
& \times R_{2} \rho(s, y(s), x(s-\tau)) d s
\end{aligned}
$$

where $\mathcal{G}^{T}(y(t))=\operatorname{col}\{g(y(t)), g(y(t-(1 / r) \sigma)), \ldots, g(y(t-$ $((r-1) / r) \sigma))\}$.

From Assumption 2 and the condition in (34), we have

$$
\begin{aligned}
\rho^{T} & (t, x(t), y(t-\sigma))\left(P_{1}+\tau R_{1}\right) \rho(t, x(t), y(t-\sigma)) \\
& \leq \ell_{1} \rho^{T}(t, x(t), y(t-\sigma)) \rho(t, x(t), y(t-\sigma)) \\
& \leq \ell_{1}\left[x^{T}(t) \mathbb{U} x(t)+y^{T}(t-\sigma) \mathbb{V} y(t-\sigma)\right] \\
\rho^{T} & (t, y(t), x(t-\tau))\left(P_{2}+\sigma R_{2}\right) \rho(t, y(t), x(t-\tau)) \\
& \leq \ell_{2} \rho^{T}(t, y(t), x(t-\tau)) \rho(t, y(t), x(t-\tau)) \\
& \leq \ell_{2}\left[y^{T}(t) \mathbb{U} y(t)+x^{T}(t-\tau) \mathbb{V} x(t-\tau)\right] .
\end{aligned}
$$

In addition, for any matrices $\mathcal{M}_{k}$ and $\mathcal{N}_{k}(k=1,2, \ldots, r)$, the following relationships hold:

$$
\begin{aligned}
2 x^{T} & \left(t-\frac{k-1}{r} \tau\right) \mathcal{M}_{k} \\
\times & {\left[x\left(t-\frac{k-1}{r} \tau\right)-x\left(t-\frac{k}{r} \tau\right)-\int_{t-\frac{k}{r} \tau}^{t-\frac{k-1}{r} \tau} \mathfrak{x}(\alpha) d \alpha\right.} \\
& \left.-\int_{t-\frac{k}{r} \tau}^{t-\frac{k-1}{r} \tau} \rho(\alpha, x(\alpha), y(\alpha-\sigma)) d \omega_{1}(\alpha)\right]=0 \\
2 y^{T} & \left(t-\frac{k-1}{r} \sigma\right) \mathcal{N}_{k} \\
\times & {\left[y\left(t-\frac{k-1}{r} \sigma\right)-y\left(t-\frac{k}{r} \sigma\right)-\int_{t-\frac{k}{r} \sigma}^{t-\frac{k-1}{r} \sigma} \mathfrak{y}(\alpha) d \alpha\right.} \\
& {\left[\int_{t-\frac{k-1}{r} \sigma}^{t} \rho(\alpha, y(\alpha), x(\alpha-\tau)) d \omega_{2}(\alpha)\right]=0 }
\end{aligned}
$$


Then, it follows from Lemma 2 that

$$
\begin{aligned}
& -2 x^{T}\left(t-\frac{k-1}{r} \tau\right) \mathcal{M}_{k} \int_{t-\frac{k}{r} \tau}^{t-\frac{k-1}{r} \tau} \mathfrak{x}(\alpha) d \alpha \\
& \leq \frac{\tau}{r} x^{T}\left(t-\frac{k-1}{r} \tau\right) \mathcal{M}_{k} S_{1}^{-1} \mathcal{M}_{k}^{T} x\left(t-\frac{k-1}{r} \tau\right) \\
& +\int_{t-\frac{k}{r} \tau}^{t-\frac{k-1}{r} \tau} \mathfrak{x}^{T}(\alpha) S_{1} \mathfrak{x}(\alpha) d \alpha \\
& -2 y^{T}\left(t-\frac{k-1}{r} \sigma\right) \mathcal{N}_{k} \int_{t-\frac{k}{r} \sigma}^{t-\frac{k-1}{r} \sigma} \mathfrak{y}(\alpha) d \alpha \\
& \leq \frac{\sigma}{r} y^{T}\left(t-\frac{k-1}{r} \sigma\right) \mathcal{N}_{k} S_{2}^{-1} \mathcal{N}_{k}^{T} y\left(t-\frac{k-1}{r} \sigma\right) \\
& +\int_{t-\frac{k}{r} \sigma}^{t-\frac{k-1}{r} \sigma} \mathfrak{y}^{T}(\alpha) S_{2} \mathfrak{y}(\alpha) d \alpha \\
& -2 x^{T}\left(t-\frac{k-1}{r} \tau\right) \mathcal{M}_{k} \\
& \times \int_{t-\frac{k}{r} \tau}^{t-\frac{k-1}{r} \tau} \rho(\alpha, x(\alpha), y(\alpha-\sigma)) d \omega_{1}(\alpha) \\
& \leq x^{T}\left(t-\frac{k-1}{r} \tau\right) \mathcal{M}_{k} R_{1}^{-1} \mathcal{M}_{k}^{T} x\left(t-\frac{k-1}{r} \tau\right) \\
& +\left(\int_{t-\frac{k}{r} \tau}^{t-\frac{k-1}{r} \tau} \rho(\alpha, x(\alpha), y(\alpha-\sigma)) d \omega_{1}(\alpha)\right)^{T} \\
& \times R_{1}\left(\int_{t-\frac{k}{r} \tau}^{t-\frac{k-1}{r} \tau} \rho(\alpha, x(\alpha), y(\alpha-\sigma)) d \omega_{1}(\alpha)\right) \\
& -2 y^{T}\left(t-\frac{k-1}{r} \sigma\right) \mathcal{N}_{k} \\
& \times \int_{t-\frac{k}{r} \sigma}^{t-\frac{k-1}{r} \sigma} \rho(\alpha, y(\alpha), x(\alpha-\tau)) d \omega_{2}(\alpha) \\
& \leq y^{T}\left(t-\frac{k-1}{r} \sigma\right) \mathcal{N}_{k} R_{2}^{-1} \mathcal{N}_{k}^{T} y\left(t-\frac{k-1}{r} \sigma\right) \\
& +\left(\int_{t-\frac{k}{r} \sigma}^{t-\frac{k-1}{r} \sigma} \rho(\alpha, y(\alpha), x(\alpha-\tau)) d \omega_{2}(\alpha)\right)^{T} \\
& \times R_{2}\left(\int_{t-\frac{k}{r} \sigma}^{t-\frac{k-1}{r} \sigma} \rho(\alpha, y(\alpha), x(\alpha-\tau)) d \omega_{2}(\alpha)\right)
\end{aligned}
$$

whereas

$$
\begin{aligned}
& \mathbb{E}\left\{\left(\int_{t-\frac{k}{r} \tau}^{t-\frac{k-1}{r} \tau} \rho(\alpha, x(\alpha), y(\alpha-\sigma)) d \omega_{1}(\alpha)\right)^{T}\right. \\
& \left.\times R_{1}\left(\int_{t-\frac{k}{r} \tau}^{t-\frac{k-1}{r} \tau} \rho(\alpha, x(\alpha), y(\alpha-\sigma)) d \omega_{1}(\alpha)\right)\right\} \\
& =\mathbb{E}\left\{\int_{t-\frac{k}{r} \tau}^{t-\frac{k-1}{r} \tau} \rho^{T}(\alpha, x(\alpha), y(\alpha-\sigma))\right. \\
& \left.\times R_{1} \rho(\alpha, x(\alpha), y(\alpha-\sigma)) d \alpha\right\} \\
& \mathbb{E}\left\{\left(\int_{t-\frac{k}{r} \sigma}^{t-\frac{k-1}{r} \sigma} \rho(\alpha, y(\alpha), x(\alpha-\tau)) d \omega_{2}(\alpha)\right)^{T}\right. \\
& \left.\times R_{2}\left(\int_{t-\frac{k}{r} \sigma}^{t-\frac{k-1}{r} \sigma} \rho(\alpha, y(\alpha), x(\alpha-\tau)) d \omega_{2}(\alpha)\right)\right\} \\
& =\mathbb{E}\left\{\int_{t-\frac{k}{r} \sigma}^{t-\frac{k-1}{r} \sigma} \rho^{T}(\alpha, y(\alpha), x(\alpha-\tau))\right. \\
& \left.\times R_{2} \rho(\alpha, y(\alpha), x(\alpha-\tau)) d \alpha\right\} \text {. }
\end{aligned}
$$

On the other hand, for $k=1,2, \ldots, r$, one can see from the sector condition in Assumption 1 that

$$
\begin{aligned}
2 g^{T} & \left(y\left(t-\frac{k-1}{r} \sigma\right)\right) \Gamma K y\left(t-\frac{k-1}{r} \sigma\right) \\
& -2 g^{T}\left(y\left(t-\frac{k-1}{r} \sigma\right)\right) \Gamma g\left(y\left(t-\frac{k-1}{r} \sigma\right)\right) \geq 0 .
\end{aligned}
$$

With the definitions in (36), for any matrices $\mathcal{T}_{1}$ and $\mathcal{T}_{2}$, we also have

$$
\begin{aligned}
2 \mathfrak{x}^{T}(t) \mathcal{T}_{1}[-A(t) x(t)+B(t) g(y(t-\sigma))-\mathfrak{x}(t)] & =0 \\
2 \mathfrak{y}^{T}(t) \mathcal{T}_{2}[-C(t) y(t)+D(t) x(t-\tau)-\mathfrak{y}(t)] & =0 .
\end{aligned}
$$

By using Lemma 2, we obtain

$$
\begin{aligned}
& -2 \mathfrak{x}^{T}(t) \mathcal{T}_{1} \triangle A(t) x(t) \leq \varepsilon_{1}^{-1} \mathfrak{x}^{T}(t) \mathcal{T}_{1} M_{1} M_{1}^{T} \\
& \quad \times \mathcal{T}_{1}^{T} \mathfrak{x}(t)+\varepsilon_{1} x^{T}(t) N_{1}^{T} N_{1} x(t) \\
& 2 \mathfrak{x}^{T}(t) \\
& \quad \mathcal{T}_{1} \triangle B(t) g(y(t-\sigma)) \leq \varepsilon_{2}^{-1} \mathfrak{x}^{T}(t) \mathcal{T}_{1} M_{1} M_{1}^{T} \\
& \quad \times \mathcal{T}_{1}^{T} \mathfrak{x}(t)+\varepsilon_{2} g^{T}(y(t-\sigma)) N_{2}^{T} N_{2} g(y(t-\sigma)) \\
& -\mathfrak{y}^{T}(t) \mathcal{T}_{2} \triangle C(t) y(t) \leq \varepsilon_{3}^{-1} \mathfrak{y}^{T}(t) \mathcal{T}_{2} M_{2} M_{2}^{T} \\
& \quad \times \mathcal{T}_{2}^{T} \mathfrak{y}(t)+\varepsilon_{3} y^{T}(t) N_{1}^{T} N_{1} y(t) \\
& 2 \mathfrak{y}^{T}(t) \mathcal{T}_{2} \triangle D(t) x(t-\tau) \leq \varepsilon_{4}^{-1} \mathfrak{y}^{T}(t) \mathcal{T}_{2} M_{2} M_{2}^{T} \\
& \quad \times \mathcal{T}_{2}^{T} \mathfrak{y}(t)+\varepsilon_{4} x^{T}(t-\tau) N_{2}^{T} N_{2} x(t-\tau) .
\end{aligned}
$$


Similarly, it can be obtained that

$$
\begin{aligned}
& -2 x^{T}(t) P_{1} \Delta A(t) x(t) \\
& \leq x^{T}(t)\left[\varepsilon_{5}^{-1} P_{1} M_{1} M_{1}^{T} P_{1}+\varepsilon_{5} N_{1}^{T} N_{1}\right] x(t) \\
& 2 x^{T}(t) P_{1} \Delta B(t) g(y(t-\sigma)) \\
& \leq \varepsilon_{6}^{-1} x^{T}(t) P_{1} M_{1} M_{1}^{T} P_{1} x(t) \\
& \quad+\varepsilon_{6} g^{T}(y(t-\sigma)) N_{2}^{T} N_{2} g(y(t-\sigma)) \\
& -2 y^{T}(t) P_{2} \Delta C(t) y(t) \\
& \leq y^{T}(t)\left[\varepsilon_{7}^{-1} P_{2} M_{2} M_{2}^{T} P_{2}+\varepsilon_{7} N_{1}^{T} N_{1}\right] y(t) \\
& 2 y^{T}(t) P_{2} \Delta D(t) x(t-\tau) \\
& \leq \varepsilon_{8}^{-1} y^{T}(t) P_{2} M_{2} M_{2}^{T} P_{2} y(t) \\
& \quad+\varepsilon_{8} x^{T}(t-\tau) N_{2}^{T} N_{2} x(t-\tau) .
\end{aligned}
$$

Taking the mathematical expectation and considering (38)-(63), one has

$$
\begin{aligned}
& \mathbb{E}\{\mathcal{L} V(t, x(t), y(t))\} \\
& \quad=\zeta(t)^{T}\left(\Xi_{1}-\Xi_{2} \Xi_{4}^{-1} \Xi_{2}^{T}-\Xi_{3} \Xi_{5}^{-1} \Xi_{3}^{T}\right) \zeta(t)
\end{aligned}
$$

where $\zeta(t)=\operatorname{col}\{\mathcal{X}(t), x(t-\tau), \mathfrak{x}(t), \mathcal{Y}(t), y(t-\sigma), \mathfrak{y}(t)$, $\mathcal{G}(y(t)), g(y(t-\sigma))\}$, and $\Xi_{i}(i=1, \ldots, 5)$ is defined in (35).

Based on the derivation conducted in Theorem 1, it follows that the uncertain stochastic model in (32) is robustly asymptotically stable in the mean square.

Remark 4: Similar to Corollary 1, we can obtain sufficient conditions ensuring the globally asymptotic stability of the genetic network in (32) without uncertain parameters. Furthermore, if there are no stochastic disturbances, we can further obtain specialized results, which are omitted here to save space. It is also worth pointing out that the main results in this paper can easily be extended to GRNs with time-varying delays by the same approach used in [26]. Note that we mainly focus on the effects brought by the norm-bounded uncertainty and the random fluctuations in this paper.

Remark 5: Lemma 2 is used to tackle the norm-bounded parameter uncertainties in the proof of Theorem 1. Comparing to existing literature, we apply the "delay-fractioning" approach and construct a more general Lyapunov functional to analyze the stability problem of the uncertain GRNs with time delays existing in both the translation process and the feedback regulation process. The novel delay-dependent conditions presented in Theorem 1 are formulated in the form of LMIs that can readily be solved by standard numerical software.

\section{NUMERICAL EXAMPLE}

In this section, two simulation examples are presented to illustrate the effectiveness of the proposed design procedures. The examples are concerned with the synthetic oscillatory network. This kind of model has theoretically been predicted and experimentally investigated as a mathematical model of the repressilator in [5].

Example 1: In the transcriptional regulators of the model mentioned above, three repressor-protein concentrations $p_{i}$ and their corresponding mRNA concentrations $m_{i}$ (where $i=l a c l$, tet $R$, or $\mathrm{cl}$ ) are considered as the continuous dynamical variables. Each of the six molecular species participates in the transcription, translation, and degradation reactions. Here, we only investigate the symmetrical case in which all three repressors

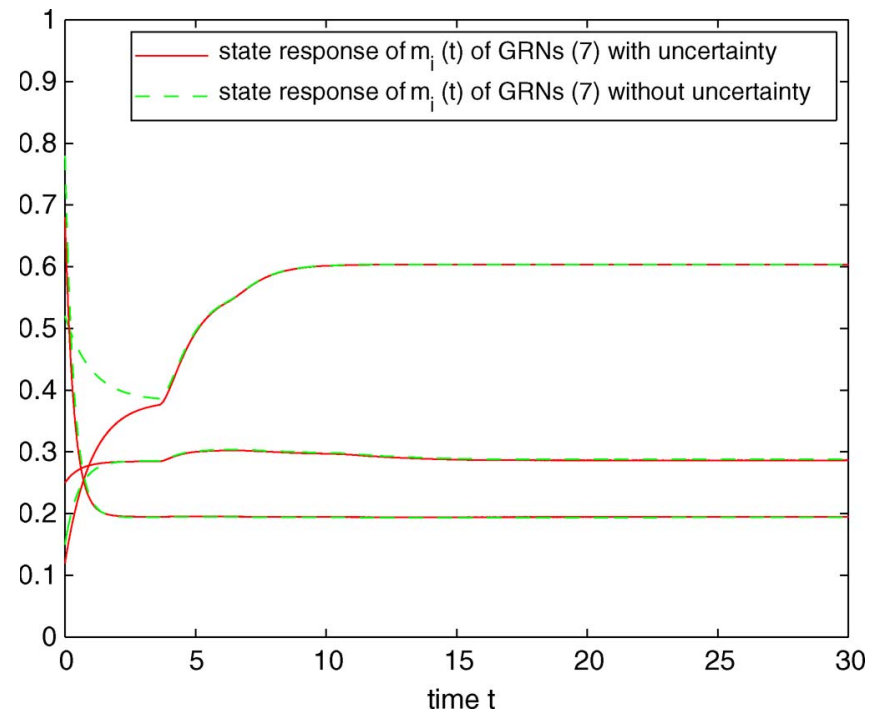

Fig. 1. mRNA concentrations.

are identical, except for their DNA-blinding specificities. By incorporating time delays and adjusting some parameters, the kinetics of the system is described by the following equations with the vector form:

$$
\begin{aligned}
\dot{m}(t) & =-A(t) m(t)+B(t) f(p(t-\sigma))+\eta \\
\dot{p}(t) & =-C(t) p(t)+D(t) m(t-\tau)
\end{aligned}
$$

where $A=\operatorname{diag}\{1,2,3\}, C=\operatorname{diag}\{2.5,2.5,2.5\}, D=\operatorname{diag}\{1$, $1,1\}$, and

$$
\begin{aligned}
B & =\left[\begin{array}{ccc}
0 & 0 & -0.6 \\
-0.6 & 0 & 0 \\
0 & -0.6 & 0
\end{array}\right] \\
M_{1} & =\left[\begin{array}{ccc}
0.1 & 0.08 & 0.04 \\
0.08 & 0.04 & -0.04 \\
0.02 & -0.06 & 0.1
\end{array}\right] \\
M_{2} & =\left[\begin{array}{ccc}
0.2 & 0.1 & -0.15 \\
0.1 & -0.3 & 0.05 \\
0.15 & -0.2 & 0.1
\end{array}\right] \\
N_{1} & =\left[\begin{array}{ccc}
0.4 & 0.1 & -0.2 \\
0.1 & 0.4 & -0.1 \\
-0.2 & -0.1 & 0.3
\end{array}\right] \\
N_{2} & =\left[\begin{array}{ccc}
0.2 & -0.3 & 0.15 \\
-0.1 & 0.2 & 0.1 \\
0.2 & -0.2 & 0.1
\end{array}\right] \\
\eta & =\left[\begin{array}{ccc}
0.6 \\
0.6 \\
0.6
\end{array}\right] .
\end{aligned}
$$

The nonlinearities are taken as $f_{j}\left(p_{j}\right)=\left(p_{j}^{2} /\left(1+p_{j}^{2}\right)\right)(j=$ $c l$, lacl, tet $R$ ), and therefore, we have $K=\operatorname{diag}\{0.65,0.65$, $0.65\}$. Furthermore, $H(t)=\operatorname{diag}\{(1 / 2) \sin (4 t), \cos (2.25 t)$, $\cos (1.25 t)\}$.

By using the Matlab LMI toolbox, it can be found that the LMIs in (12) are feasible. When we set $r=1$, the time delays can be achieved as $\tau=2.5$, and $\sigma=3.6$. The simulation results of the trajectories of $m_{i}(t), p_{i}(t)$ are shown in Figs. 1 and 2, 


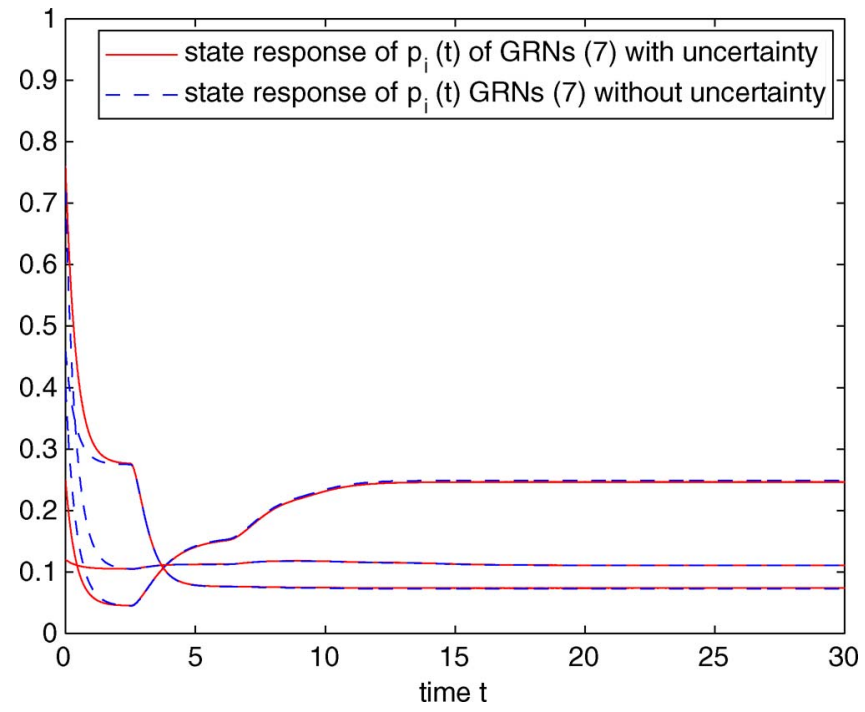

Fig. 2. Protein concentrations.

where the different initial states for system in (1) are taken as $[0,1] \times[0,1]$. The simulation results further indicate that the given GRN with time delays and uncertain parameters is asymptotically robustly stable.

Example 2: In this example, we consider the uncertain genetic networks in (32) with noise disturbances with $\tau=2$ and $\sigma=3$, in which there are five nodes denoting the regulation factors, and the designed parameters in (32) are given by $A=$ $\operatorname{diag}\{1,2,3,4,5\}, C=2.5 I$, and $D=2 I$, and the coupling matrix is given as

$$
B=0.8 \times\left[\begin{array}{ccccc}
0 & 0 & -1 & 0 & 0 \\
-1 & 0 & 0 & 0 & 0 \\
0 & -1 & 0 & 0 & 0 \\
0 & 0 & -1 & 0 & 0 \\
0 & 0 & 0 & 0 & -1
\end{array}\right]
$$

where the coefficient 0.8 is the transcriptional rate. The parameter uncertainties satisfy $M_{l}=N_{l}=0.25 I(l=1,2)$ and $H(t)=\operatorname{diag}\{\sin (2 t), \cos (5 t), \cos (1.5 t), 0.5 \sin (4 t), \cos (4.25 t)\}$. The noise intensity vectors satisfy Assumption 2 with $\mathbb{U}=$ $\mathbb{V}=0.5 I$. The nonlinear function is given by $f_{i}(s)=s^{2} /(1+$ $\left.s^{2}\right)(i=1,2, \ldots, 5)$, i.e., $K=0.65 I$.

By using the Matlab LMI toolbox, we can find that the LMIs in (34) and (35) are feasible (the solutions are not given here for the purpose of space saving). When we set $r=1$, the numerical simulation results are given in Figs. 3 and 4, with the initial states randomly taken in $[0,1] \times[0,1]$, which further implies that the uncertain GRN with noises perturbations is globally robustly asymptotically stable in the mean square.

\section{CONCLUSION}

In this paper, we have dealt with the robust stability analysis problem for GRNs with time delays, norm-bounded parameter uncertainties, and state-dependent Brownian motions. By using a Lyapunov functional approach, stochastic analysis tools, and the LMI technique, we have constructed a novel Lyapunov-Krasovskii functional and then derived sufficient conditions in terms of LMIs to ensure globally asymptotically

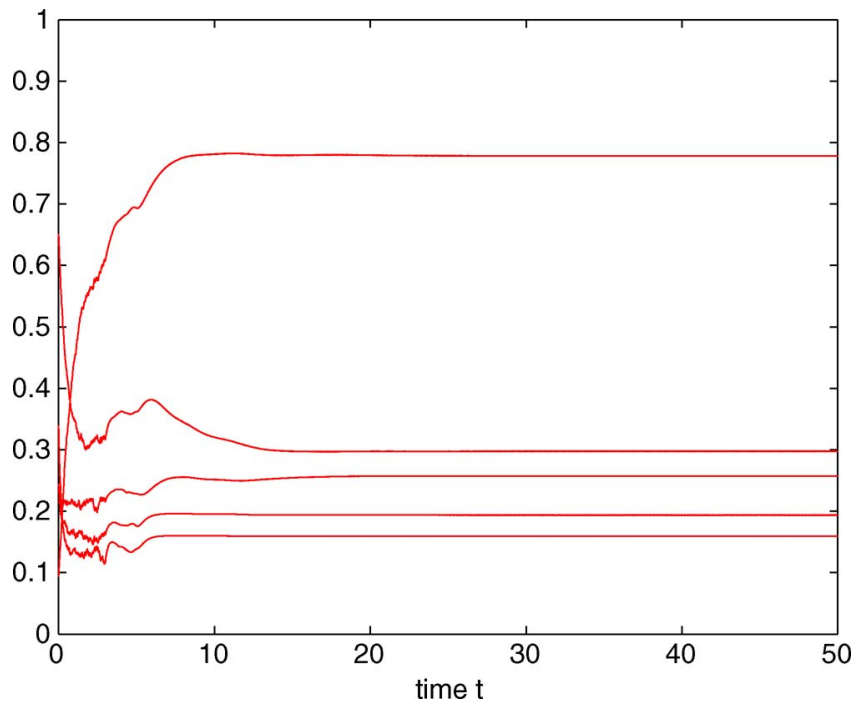

Fig. 3. State response of $m(t)$ of GRNs [see (32)].

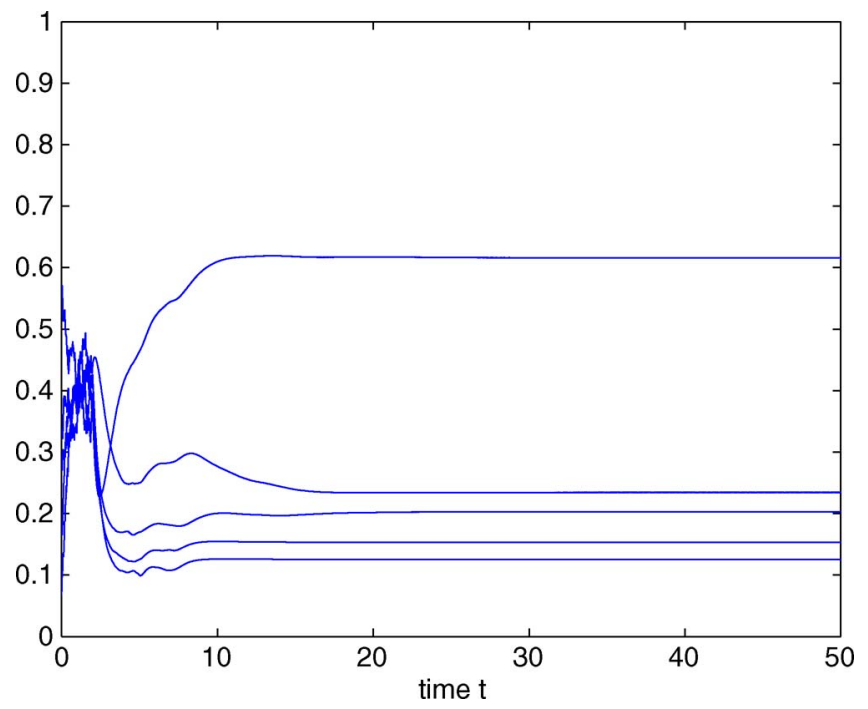

Fig. 4. State response of $p(t)$ of GRNs [see (32)].

robust stability of the addressed delayed uncertain genetic networks. Moreover, the LMI-based criteria can readily be verified by using standard numerical software. An important feature of the results reported here is that the stability condition is dependent on the upper bounds of the time delays, which is made possible by utilizing the most updated techniques for achieving delay dependence. To the best of our knowledge, the present research represents the first attempt to develop a novel computational approach specifically for the robust stability of uncertain GRNs with or without noise perturbations. In the end of this paper, two simulation examples have been exploited to illustrate the applicability and usefulness of the developed theoretical results.

\section{REFERENCES}

[1] X. Cai and X. Wang, "Stochastic modeling and simulation of gene networks," IEEE Signal Process. Mag., vol. 24, no. 1, pp. 27-36, Jan. 2007.

[2] G. Chesi and Y. S. Hung, "Stability analysis of uncertain genetic sum regulatory networks," Automatica, vol. 44, no. 9, pp. 2298-2305, Sep. 2008. 
[3] L. Chen and K. Aihara, "Stability of genetic regulatory networks with time delay," IEEE Trans. Circuits Syst. I, Fundam. Theory Appl., vol. 49, no. 5, pp. 602-609, May 2002.

[4] B. Chen and P. Chen, "Robust engineered circuit design principles for stochastic biochemical networks with parameter uncertainties and disturbances," IEEE Trans. Biomed. Circuits Syst., vol. 2, no. 2, pp. 114-132, Jun. 2008.

[5] M. B. Elowitz and S. Leibler, "A synthetic oscillatory network of transcriptional regulators," Nature, vol. 403, no. 6767, pp. 335-338, Jan. 2000.

[6] K. Q. Gu, V. L. Kharitonov, and J. Chen, Stability of Time-Delay Systems. Boston, MA: Birkhauser, 2003.

[7] H. de Jong, J. Geiselmann, C. Hernandez, and M. Page, "Genetic network analyzer: Qualitative simulation of genetic regulatory networks," Bioinformatics, vol. 19, no. 3, pp. 336-344, Feb. 2003.

[8] H. de Jong and R. Lima, "Modeling the dynamics of genetic regulatory networks: Continuous and discrete approaches," Lect. Notes Phys., vol. 671 , pp. 307-340, 2005.

[9] H. Gao, J. Lam, and G. Chen, "New criteria for synchronization stability of general complex dynamical networks with coupling delays," Phys. Lett. A, vol. 360, no. 2, pp. 263-273, Dec. 2006.

[10] H. Gao and T. Chen, "Network-based $\mathcal{H}_{\infty}$ output tracking control," IEEE Trans. Autom. Control, vol. 53, no. 3, pp. 655-667, Apr. 2008.

[11] H. Kushur, Stochastic Stability and Control. New York: Academic, 1976.

[12] J. Lam, H. Gao, and C. Wang, "Stability analysis for continuous systems with two additive time-varying delay components," Syst. Control Lett., vol. 56, no. 1, pp. 16-24, Jan. 2007.

[13] C. Li, L. Chen, and K. Aihara, "Stability of genetic networks with SUM regulatory logic: Lur'e system and LMI approach," IEEE Trans. Circuits Syst. I, Reg. Papers, vol. 53, no. 11, pp. 2451-2458, Nov. 2006.

[14] C. Li, L. Chen, and K. Aihara, "Stochastic stability of genetic networks with disturbance attenuation," IEEE Trans. Circuits Syst. II, Exp. Briefs, vol. 54, no. 10, pp. 892-896, Oct. 2007.

[15] Y. Liu, Z. Wang, and X. Liu, "Global exponential stability of generalized recurrent neural networks with discrete and distributed delays," Neural Netw., vol. 19, no. 5, pp. 667-675, Jun. 2006.

[16] S. Mou, H. Gao, W. Qiang, and K. Chen, "New delay-dependent exponential stability for neural networks with time delay," IEEE Trans. Syst., Man, Cybern. B, Cybern., vol. 38, no. 2, pp. 571-576, Apr. 2008.

[17] S. Mou, Y. Zhao, H. Gao, and W. Qiang, "Further improvement on synchronization stability of complex networks with coupling delays," Int. J. Comput. Math., vol. 85, no. 8, pp. 1255-1263, Aug. 2008.

[18] J. Paulsson, "Summing up the noise in gene networks," Nature, vol. 427, no. 6973, pp. 415-418, Jan. 2004.

[19] D. Peaucelle, D. Arzelier, D. Henrion, and F. Gouaisbaut, "Quadratic separation for feedback connection of an uncertain matrix and an implicit linear transformation," Automatica, vol. 43, no. 5, pp. 795-804, May 2007.

[20] F. Ren and J. Cao, "Asymptotic and robust stability of genetic regulatory networks with time-varying delays," Neurocomputing, vol. 71, no. 4-6, pp. 834-842, Jan. 2008.

[21] E. Someren, L. Backer, and M. Reinders, "Genetic network modeling," Lect. Notes Phys., vol. 671, pp. 307-340, 2005.

[22] V. Shahrezaei and P. Swain, "The stochastic nature of biochemical networks," Curr. Opin. Biotechnol., vol. 19, no. 4, pp. 369-374, Aug. 2008.

[23] T. Tian, K. Burrage, P. M. Burrage, and M. Carletti, "Stochastic delay differential equations for genetic regulatory networks," J. Comput. Appl. Math., vol. 205, no. 2, pp. 696-707, Aug. 2007.

[24] G. Wang and J. Cao, "Robust exponential stability analysis for stochastic genetic networks with uncertain parameters," Commun. Nonlinear Sci. Numer. Simul., vol. 14, no. 8, pp. 3369-3378, Aug. 2009.

[25] Y. Wang, Z. Wang, and J. Liang, "A delay fractioning approach to global synchronization of delayed complex networks with stochastic disturbances," Phys. Lett. A, vol. 372, no. 39, pp. 6066-6073, Sep. 2008.

[26] Y. Wang, Z. Wang, and J. Liang, "Global synchronization for delayed complex networks with randomly occurring nonlinearities and multiple stochastic disturbances," J. Phys. A, Math. Theor, vol. 42, no. 13, p. 135 101, Apr. 2009.

[27] Z. Wang, F. Yang, D. W. C. Ho, S. Swift, A. Tucker, and X. Liu, "Stochastic dynamic modeling of short gene expression time-series data," IEEE Trans. Nanobiosci., vol. 7, no. 1, pp. 44-55, Mar. 2008.

[28] Z. Wang, J. Lam, G. Wei, K. Fraser, and X. Liu, "Filtering for nonlinear genetic regulatory networks with stochastic disturbances," IEEE Trans. Autom. Control, vol. 53, no. 10, pp. 2448-2457, Nov. 2008.

[29] Z. Wang, H. Gao, J. Cao, and X. Liu, "On delayed genetic regulatory networks with polytopic uncertainties: Robust stability analysis," IEEE Trans. Nanobiosci., vol. 7, no. 2, pp. 154-163, Jun. 2008.

[30] Z. Wang, H. Shu, J. Fang, and X. Liu, "Robust stability for stochastic Hopfield neural networks with time delays," Nonlinear Anal.: Real World Appl., vol. 7, no. 5, pp. 1119-1128, Dec. 2006.

[31] Z. Wang, B. Huang, and H. Unbehauen, "Robust $H_{\infty}$ observer design of linear time-delay systems with parametric uncertainty," Syst. Control Lett., vol. 42, no. 4, pp. 303-312, Apr. 2001.

[32] V. Wolf, "Modelling of biochemical reactions by stochastic automata networks," Electron. Notes Theor. Comput. Sci., vol. 171, no. 2, pp. $197-$ 208, Jul. 2007.

[33] R. Yang, H. Gao, and P. Shi, "Novel robust stability criteria for stochastic Hopfield neural networks with time delays," IEEE Trans. Syst., Man, Cybern. B, Cybern., vol. 39, no. 2, pp. 467-474, Apr. 2009.

[34] F. Zhang, The Schur Complement and its Applications. New York: Springer-Verlag, 2005.

[35] Y. Zhao, H. Gao, J. Lam, and B. Du, "Stability and stabilization of delayed T-S fuzzy systems: A delay partitioning approach," IEEE Trans. Fuzzy Syst., vol. 17, no. 4, pp. 750-762, 2009.

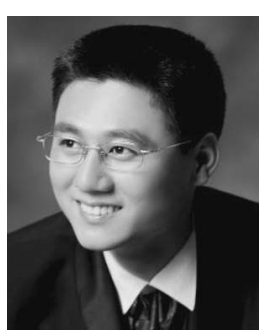

Yao Wang received the B.Sc. and M.Sc. degrees in applied mathematics from Yangzhou University, Yangzhou, China, in 2004 and 2007, respectively. He is currently working toward the Ph.D. degree with the School of Information Science and Technology, Donghua University, Shanghai, China.

His research interests include dynamical systems, complex networks, systems biology, stochastic systems, and time-delay systems. He is a very active reviewer for many international journals.

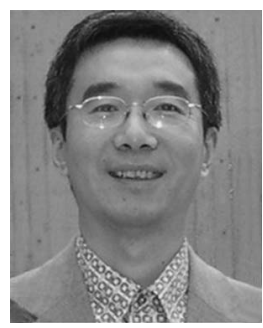

Zidong Wang (SM'03) was born in Jiangsu, China, in 1966. He received the B.Sc. degree in mathematics from Suzhou University, Suzhou, China, in 1986 and the M.Sc. degree in applied mathematics and the $\mathrm{Ph} . \mathrm{D}$. degree in electrical and computer engineering from Nanjing University of Science and Technology, Nanjing, China, in 1990 and 1994, respectively.

$\mathrm{He}$ is currently a Professor of dynamical systems and computing with Brunel University, Uxbridge, U.K. He has published more than 120 papers in refereed international journals. His research interests include dynamical systems, signal processing, bioinformatics, control theory, and applications.

Dr. Wang is currently serving as an Associate Editor for 12 international journals, including IEEE TRANSACTIONS ON AUTOMATIC CONTROL, IEEE TRansactions on NEURAL NETworks, IEEE Transactions on Signal Processing, IEEE Transactions ON Systems, Man, and CYBERNETICS-PART C, and IEEE TRANSACTIONS ON CONTROL SYSTEMS TECHNOLOGY.

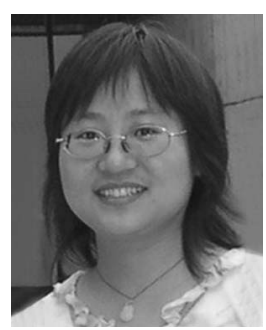

Jinling Liang received the B.Sc. and M.Sc. degrees in mathematics from Northwest University, Xi'an, China, in 1997 and 1999, respectively, and the Ph.D. degree in applied mathematics from Southeast University, Nanjing, China, in 2006.

From January 2004 to March 2004 and from March 2009 to August 2009, she was a Research Assistant with the University of Hong Kong, Pok Fu Lam, Hong Kong. From March to April 2004, she was a Research Assistant with the City University of Hong Kong, Kowloon, Hong Kong. From April 2007 to March 2008, she was a Postdoctoral Research Fellow with the Department of Information Systems and Computing, Brunel University, Uxbridge, U.K., where she was sponsored by the Royal Society Sino-British Fellowship Trust Award of the U.K. She is currently an Associate Professor with the Department of Mathematics, Southeast University. She has published around 20 papers in refereed international journals. She is currently serving as an Associate Editor for Neurocoomputing. Her research interests include neural networks, complex networks, nonlinear systems, and bioinformatics.

Dr. Liang is a member of the program committee for some international conferences and serves as a very active reviewer for many international journals. 